\title{
A space-time random field model for electricity forward prices
}

\author{
Fred Espen Benth* $\quad$ Florentina Paraschiv*†
}

March 27, 2017

\begin{abstract}
Stochastic models for forward electricity prices are of great relevance nowadays, given the major structural changes in the market due to the increase of renewable energy in the production mix. In this study, we derive a spatio-temporal dynamical model based on the Heath-Jarrow-Morton (HJM) approach under the Musiela parametrization, which ensures an arbitrage-free model for electricity forward prices. The model is fitted to a unique data set of historical price forward curves. As a particular feature of the model, we disentangle the temporal from spatial (maturity) effects on the dynamics of forward prices, and shed light on the statistical properties of risk premia, of the noise volatility term structure and of the spatio-temporal noise correlation structures. We find that the short-term risk premia oscillates around zero, but becomes negative in the long run. We identify the Samuelson effect in the volatility term structure and volatility bumps, explained by market fundamentals. Furthermore we find evidence for coloured noise and correlated residuals, which we model by a Hilbert space-valued normal inverse Gaussian Lévy process with a suitable covariance functional.
\end{abstract}

JEL Classification: C02, C13, C23

Keywords: spatio-temporal models, price forward curves, term structure volatility, risk premia, electricity markets

*Department of Mathematics, University of Oslo, PO Box 1053 Blindern, N-0316 Oslo, Norway, fredb@math.uio.no, Fax: +47 22854349.

$\dagger *$ Corresponding author: Florentina Paraschiv, NTNU Business School, Norwegian University of Science and Technology, 7491 Trondheim, florentina.paraschiv@ntnu.no and University of St. Gallen, Institute for Operations Research and Computational Finance, Bodanstrasse 6, CH-9000 St. Gallen, Switzerland 


\section{Introduction}

2 There exist two main approaches for modelling forward prices in commodity and en-

3 ergy markets. The classical way goes by specifying a stochastic model for the spot 4 price, and from this model derive the dynamics of forward prices based on no-arbitrage 5 principles (see Lucia and Schwartz (2002), Cartea and Figueroa (2005), Roncoroni and

6 Geman (2006), Benth, Kallsen, and Meyer-Brandis (2007), Garcia, Klüppelberg, and 7 Müller (2011), Barndorff-Nielsen, Benth, and Veraart (2013), Weron and Zator (2014), 8 and Benth, Klüppelberg, Müller, and Vos (2014)). The alternative is to follow the Heath9 Jarrow-Morton approach and to specify the dynamics of the forward prices directly, as it 10 has been done in Roncoroni and Guiotto (2001), Benth and Koekebakker (2008), Weron 11 and Borak (2008) and Kiesel, Schindlmayr, and Boerger (2009). All these studies model 12 the forward prices using multifactor models driven by Brownian motion. However, em13 pirical findings in Koekebakker and Ollmar (2005), Frestad (2008) suggest that there is 14 a substantial amount of variation in forward prices which cannot be explained by a few

common factors. Furthermore, the models that directly specify the dynamics of forward contracts ignore the fact that the returns of forward prices in electricity markets are far from being Gaussian distributed and have possible stochastic volatility effects.

The idea of modeling power forward prices with a random field model goes back to Audet, Heiskanen, Keppo, and Vehviläinen (2004), who studied theoretically a Gaussian model with certain mean-reversion characteristics. Their modelling framework is closely related to Kennedy (1994) and Goldstein (2000) who proposed random field models for the term structure of interest rates. Random-field models for forward prices in power markets have been explored statistically and mathematically by Andresen, Koekebakker, and Westgaard (2010). There the authors model electricity forwards returns for different 25 times to maturity using a multivariate normal inverse Gaussian (NIG) distribution to capture the idiosyncratic risk and heavy tails behavior and conclude the superiority of this approach versus Gaussian-based multifactor models in terms of goodness of fit. Their 
analysis seems to be based on the assumption that forward prices follow an exponential spatio-temporal stochastic process. When modeling forward prices evolving along time to maturity, the so-called Musiela parametrization, rather than time at maturity, one must be careful with how the time to maturity affects a price change. Indeed, in this so-called Musiela parametrization context of forward prices an additional drift term must be added to the dynamics to preserve arbitrage-freeness of the model. .

In this paper we propose to model the forward price dynamics by a spatio-temporal random field based on the Heath-Jarrow-Morton (HJM) approach under the Musiela parametrization (see Heath, Jarrow, and Morton (1992)), which ensures an arbitragefree dynamics. After discretizing the model in time and space, we can separate seasonal features in the risk premium and random perturbations of the prices, and apply this to obtain information of the statistical characteristics of the data. Our model formulation disentangles typical components of forward prices such as: the deterministic seasonality pattern and the stochastic component including the market price of risk and the noise. We show the importance of rigourously modeling each component in the context of an empirical application to electricity forward prices, in which a unique panel data set of 2'386 hourly price forward curves is employed for the German electricity index PHELIX. The index is generated each day for a horizon of 6 years, ranging from 01/01/2009 until $15 / 07 / 2015$. Each day a new price forward curve (PFC) is generated based on the newest information from current futures prices observed at EPEX円

The dynamics of price forward curves (PFCs) are modeled with respect to two dimensions: temporal and spatial (the space dimension here refers to time to maturity of the forward). In particular, the changes in the level of a PFC for one specific maturity point between consecutive days reflect two features:

Firstly, as time passes, dynamics in time of on-going futures prices with a certain delivery period reflect changes in the market expectation. In particular, maturing futures

\footnotetext{
${ }^{1}$ Electricity for delivery on the next day is traded at the European Power Exchange (EPEX SPOT) in
} Paris. 
are replaced by new ones in the market ${ }^{2}$ Changes in the market expectations reflect updates in weather forecasts, planned outages due to maintenance of power plants, energy policy announcements or expected market structural changes. Germany adopted the Renewable Energy Act (EEG) in 2000, accordingly to which producers of renewable energies (wind, photovoltaic etc.) receive a guaranteed compensation (technology dependent feed-in tariffs). Renewable energies are fed with priority into the grid, replacing thus in production other traditional fuels (oil, gas, coal). Given the difficulty of getting accurate weather forecasts, electricity demand/supply disequilibria became more frequent, which increased the volatility of electricity prices. Furthermore, it has been empirically shown that due to the low marginal production costs of wind and photovoltaic, the general level of electricity prices decreased over time (see Paraschiv, Erni, and Pietsch (2014)), which explains the shift in time of the general level of the analyzed PFCs.

Secondly, as time passes, the time to maturity of one specific product decreases and maturing futures are replaced by new ones in the market. In the German electricity market, weekly, monthly, quarterly and yearly futures are traded. Given the small number of different exchange-traded futures, and thus different maturities, the stochastic component of the (deseasonalized) PFCs shows a typical step-wise pattern when depicted graphically. Indeed, the different futures prices are represented as vertical lines over their respective delivery periods, with the hight of the lines being the prices. Hence, the change in the level of the PFC over a time step is impacted through a change in the market expectations as well as a change in time to maturity. Both effects are displayed in Figure 1.

Our proposed model is fitted to the generated PFCs. We first perform a deseasonalization of the initial curves, where the seasonal component takes into account typical patterns observed in electricity prices (see Paraschiv (2013) and Paraschiv, Fleten, and Schürle (2015)). The stochastic component of the deseasonalized forward curves will consist of a risk premium and residual noise, where the risk premium is assumed to be

\footnotetext{
${ }^{2}$ In the German electricity market, weekly, monthly, quarterly or yearly futures are traded.
} 


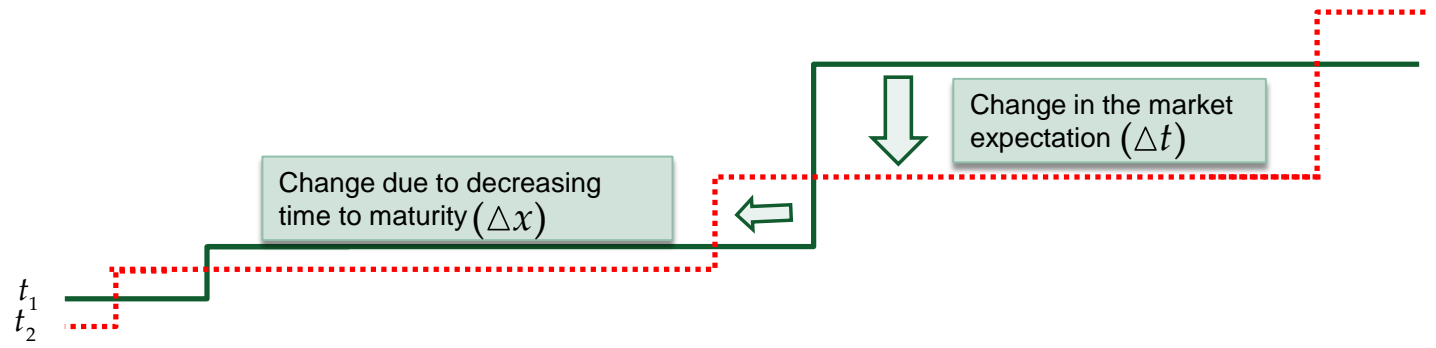

Figure 1: The effect of time and maturity change on the dynamics of forward prices.

proportional to the deseasonalized forward price by a term structure of market prices of risk. We estimate the market price of risk and examine the distribution of the residual noise volatility and its spatio-temporal correlations structures. Our results show that the short-term risk premia oscillates around zero, but becomes negative in the long run, which is consistent with the empirical literature (Burger, Graeber, and Schindlmayr (2007)). The descriptive statistics of the noise marginals reveals clear evidence for a coloured-noise with leptokurtic distribution and heavy-tails, which we suggest to model by a normal inverse Gaussian distribution (NIG) $]^{3}$ We further examine the term structure of volatility where we are able to identify the Samuelson effect and volatility bumps. The occurrence of volatility bumps are explained by the trading activity in the market for futures of specific maturities (delivery periods). The spatial correlation structure of the noise is stationary with a fast-decaying pattern: decreasing correlations with increased distance between maturity points along one curve.

Based on the empirical evidence, we further stylize our model and specify a spatiotemporal mathematical formulation for the coloured noise time series. After explaining the Samuelson effect in the volatility term structure, the residuals are modeled by a NIG Lévy

${ }^{3}$ Similar results can be found in Frestad, Benth, and Koekebakker (2010), who analyzed the distribution of daily $\log$ returns of individual forward contracts at Nord Pool and found that the univariate NIG distribution performed best in fitting the return data. 
process with values in a convenient Hilbert space, which allows for a natural formulation of a covariance functional. We model, in this way, the typical fat tails and fast-decaying pattern of spatial correlations. Our modeling approach contributes in several ways beyond that of Andresen, Koekebakker, and Westgaard (2010): we disentangle the temporal from spatial (maturity) effects on the dynamics of forward prices honouring the no-arbitrage condition. This provides us with a data set in time and space where we can reveal and analyse the statistical properties of risk premia, of the noise volatility term structure and of the spatio-temporal noise correlation structures. Moreover, we introduce a mathematical framework for modelling the forward price dynamics which links to the empirics, including the Samuelson effect, the correlation structure along maturities and non-Gaussian price residuals. In conclusion, we formulate an arbitrage-free random field model for the power forward price dynamics in space and time which honours the statistical findings.

A mathematical treatise of the more general random field models of HJM type as we propose in this paper can be found in Benth and Krühner (2014). The issue of pricing derivatives for such random field models is discussed in Benth and Krühner (2015), while Benth and Lempa (2014) analyse portfolio strategies in energy markets with infinite dimensional noise. Our proposed forward price dynamics is thus suitable for further applications to both derivatives pricing and risk management. Efficient numerical approaches for simulation are also available, see for example Barth and Benth (2014). Thus, exotic energy derivatives may be priced by Monte Carlo simulations from the model. One may also simulate scenarios for hedges and portfolio positions in energy forwards. The flexibility and practical applicability of our proposed space-time random field dynamics makes it accessible for stress testing with other, competing models. For example, many in-house forward price models are based on multi-factor spot price dynamics. One may compare investment decisions in the two models, as well as analyse robustness of valuation of derivatives prices. Ambit fields is an alternative class of random fields which can be used for dynamic modeling of forward prices in power markets, see Barndorff-Nielsen, Benth, 
and Veraart (2014). In Barndorff-Nielsen, Benth, and Veraart (2015) and Benth and Krühner (2015), infinite-dimensional cross-commodity forward price models are proposed and analysed.

The rest of the paper is organized as follows: In section 2 we present the mathematical formulation of the spatio-temporal random field model. In sections 3 and 4 we describe the data used for the application and present descriptive statistics on the risk premia, volatility, correlations and noise. The estimation results are shown in section 5 , and in section 6 we specify a mathematical model for the residuals based on the statistical findings. Finally, section 7 concludes.

\section{Spatio-temporal random field modeling of forward prices}

The Heath-Jarrow-Morton (HJM) approach (see Heath, Jarrow, and Morton (1992)) has been advocated as an attractive modelling framework for energy and commodity forward prices (see Benth, Šaltytė Benth, and Koekebakker (2008), Benth and Krühner (2014), Benth and Krühner (2015), Benth and Koekebakker (2008), Clewlow and Strickland $(2000)$ ). If $F_{t}(T)$ denotes the forward price at time $t \geq 0$ for delivery of a commodity at time $T \geq t$, we introduce the so-called Musiela parametrization $x=T-t$ and let $G_{t}(x)$ be the forward price for a contract with time to maturity $x \geq 0$. The graphical representation in Figure 2 shows comparatively the difference between thinking in terms of "time at maturity", $T$, versus "time to maturity" $x$. Note that $G_{t}(x)=F_{t}(t+x)$. It is known (see e.g., Benth and Krühner (2014) and Benth and Krühner (2015)) that the stochastic process $t \mapsto G_{t}(x), t \geq 0$ is the solution of a stochastic partial differential equation (SPDE),

$$
d G_{t}(x)=\left(\partial_{x} G_{t}(x)+\beta(t, x)\right) d t+d W_{t}(x)
$$



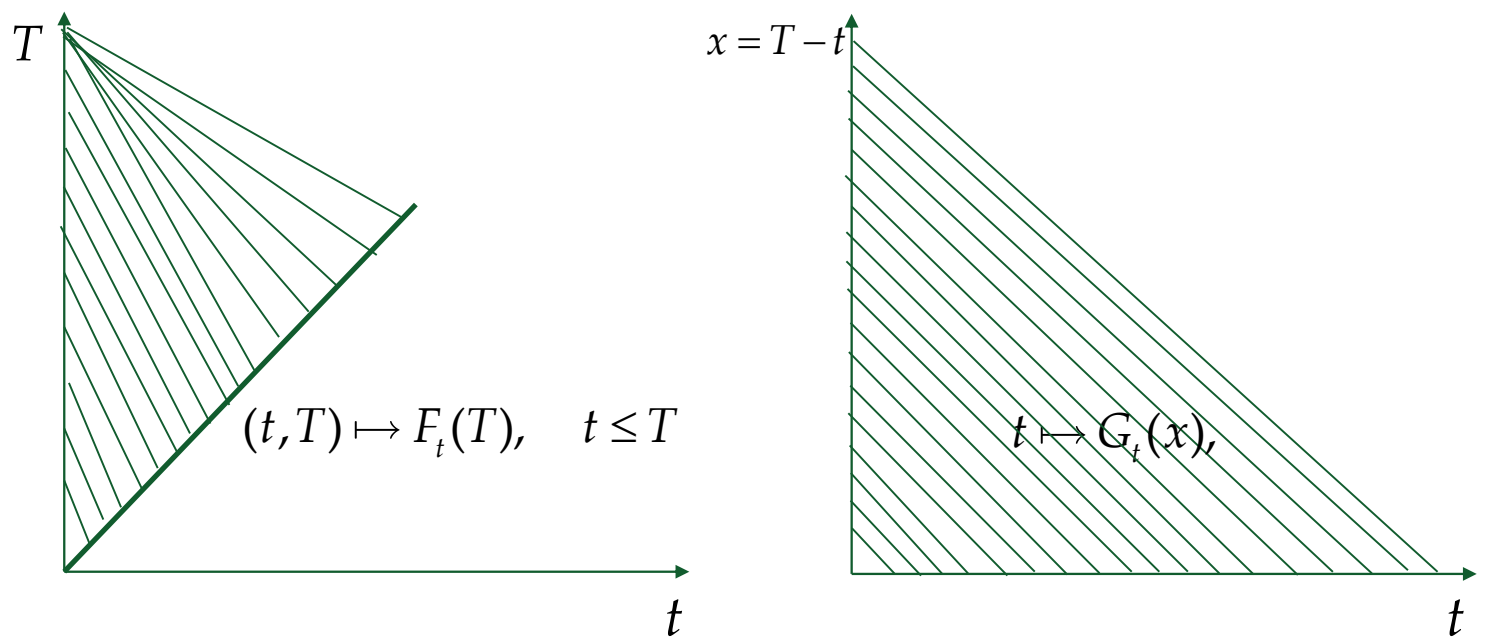

Figure 2: Theoretical model: time at maturity (first graph) versus time to maturity (second graph).

where $\partial_{x}=\partial / \partial x$ is the differential operator with respect to time to maturity $x, \beta$ is a spatio-temporal process modelling the market price of risk and finally $W$ is a spatiotemporal random field which describes the randomly evolving residuals in the dynamics.

To make the model for the forward price dynamics $G$ rigorous, it has to be formulated as a stochastic process in time, taking values in a space of curves on the positive real line $\mathbb{R}_{+}$. By a curve on $\mathbb{R}_{+}$, we understand the graph of a function $x \mapsto f(x)$, where $x \in \mathbb{R}_{+}$. It would be more precise to talk of functions rather than curves on $\mathbb{R}_{+}$, but we want to preserve the analogy to the frequently used notion of forward curves. Typically, this space of curves is endowed with a Hilbert space structure. Denoting this Hilbert space of curves by $\mathcal{H}$, the SPDE (1) is interpreted as a stochastic differential equation in $\mathcal{H}$. Moreover, the $\mathcal{H}$-valued process $W_{t}$ is a martingale, and encodes a correlation structure in space and time for the forward prices, as well as the distribution of price increments at fixed times to maturity $x$ and the term structure of volatility. The latter includes the Samuelson effect, which is predominant in commodity markets where stationarity of 
prices is an empirical characteristic. We refer to Benth and Krühner (2015) for a rigorous mathematical description and analysis of (1) in the Hilbert space framework, where a specific example of an appropriate space of curves $\mathcal{H}$ suitable for commodity markets is proposed.

In this paper we will analyse a discrete-time version of the process $G_{t}$, obtained from an Euler discretization of (1). In particular, our focus will be on an analysis of the seasonal structure, the market price of risk and finally the probabilistic features of the noise component $W_{t}$. To this end, suppose that

$$
G_{t}(x)=f_{t}(x)+s_{t}(x),
$$

where $s_{t}(x)$ is a deterministic seasonality function. We assume that $\mathbb{R}_{+}^{2} \ni(t, x) \mapsto s_{t}(x) \in$ $\mathbb{R}$ is a bounded and measurable function, typically positive. Note that if we construct the seasonality function from a spot price model, then naturally $s_{t}(x)=s(t+x)$, where $s$ is the seasonality function of the commodity spot price (see Benth, Šaltytè Benth, and Koekebakker (2008)). Indeed, it is reasonable that a seasonality function should depend on the actual maturity date (i.e., $t+x=T$ ), which points to a specification where $s_{t}(x):=s(t+x)$ also in the general case. Motivated by (1), we furthermore assume that the deseasonalized forward price curve, denoted by $f_{t}(x)$, has the dynamics

$$
d f_{t}(x)=\left(\partial_{x} f_{t}(x)+\theta(x) f_{t}(x)\right) d t+d W_{t}(x),
$$

with $\mathbb{R}_{+} \ni x \mapsto \theta(x) \in \mathbb{R}$ is a bounded and measurable function modeling the risk premium. Hence, we suppose that the risk premium is proportional to the deseasonalized forward price, with proportionality varying with time to maturity. With this definition, 
we note that:

$$
\begin{aligned}
d G_{t}(x) & =d f_{t}(x)+d s_{t}(x) \\
& =\left(\partial_{x} f_{t}(x)+\theta(x) f_{t}(x)\right) d t+\partial_{t} s_{t}(x) d t+d W_{t}(x) \\
& =\left(\partial_{x} G_{t}(x)+\left(\partial_{t} s_{t}(x)-\partial_{x} s_{t}(x)\right)+\theta(x)\left(G_{t}(x)-s_{t}(x)\right)\right) d t+d W_{t}(x) .
\end{aligned}
$$

As indicated above, naturally $s_{t}(x)=s(t+x)$, and hence $\partial_{t} s_{t}(x)=\partial_{x} s_{t}(x)$. Therefore, we see that $G_{t}(x)$ satisfies (1) with $\beta(t, x):=\theta(x) f_{t}(x)$, i.e., that the market price of risk is proportional to the deseasonalized forward prices. Note that we have implicitly assumed differentiability of $s_{t}(x)$ in the above derivation.

Let us next discretize the dynamics of $f_{t}$ in (3), in order to obtain a time series dynamics of the (deseasonalized) forward price curve. Let $\left\{x_{1}, \ldots, x_{N}\right\}$ be a set of equidistant time-to-maturity dates with resolution $\Delta x:=x_{i}-x_{i-1}$ for $i=2, \ldots, N$. At time $t=\Delta t, \ldots, M \Delta t$, where $M \Delta t=T$ for some terminal time $T$, we observe for each time-to-maturity date $x \in\left\{x_{1}, \ldots, x_{N}\right\}$ a point on the price-forward curve $G_{t}(x)$ and a corresponding point on the seasonality curve $s_{t}(x)$. A standard approximation of the derivative operator $\partial_{x}$ is

$$
\partial_{x} f_{t}(x) \approx \frac{f_{t}(x+\Delta x)-f_{t}(x)}{\Delta x}
$$

Next, after doing an Euler discretization in time of (3), we obtain the time series approximation for $f_{t}(x)$. With $x \in\left\{x_{1}, \ldots, x_{N}\right\}$ and $t=\Delta t, \ldots,(M-1) \Delta t$,

$$
f_{t+\Delta t}(x)=\left(f_{t}(x)+\frac{\Delta t}{\Delta x}\left(f_{t}(x+\Delta x)-f_{t}(x)\right)+\theta(x) f_{t}(x) \Delta t+\epsilon_{t}(x)\right.
$$

where $\epsilon_{t}(x):=W_{t+\Delta t}(x)-W_{t}(x)$. We define the time series $Z_{t}(x)$ for $x \in\left\{x_{1}, \ldots, x_{N}\right\}$ and $t=\Delta t, \ldots,(M-1) \Delta t$

$$
Z_{t}(x):=f_{t+\Delta t}(x)-f_{t}(x)-\frac{\Delta t}{\Delta x}\left(f_{t}(x+\Delta x)-f_{t}(x)\right)
$$


which implies

$$
Z_{t}(x)=\theta(x) f_{t}(x) \Delta t+\epsilon_{t}(x),
$$

where changes between the stochastic components of forward curves incorporate risk premia and changes in the noise. Since we are interested in analysing the properties of the noise volatility, to account for Samuelson effect in forward prices, the model residuals $\epsilon_{t}(x)$ are further decomposed in:

$$
\epsilon_{t}(x)=\sigma(x) \tilde{\epsilon}_{t}(x)
$$

where $\tilde{\epsilon}_{t}(x)$ are the standardized residuals.

The time series model (6) will be our object of study in this paper, where we are concerned with inference of the market price of risk proportionality factor $\theta(x)$ and the probabilistic structure of $\tilde{\epsilon}_{t}(x)$. Since our concern is power markets, we aim at a (time and space) discrete curve $Z_{t}(x)$ from forward prices over a delivery period. How to recover data for $Z$ in such markets will be discussed in the next section. We remark here that we will choose a procedure of constructing a seasonal function which provides information on $s_{t}(x)$ at discrete time and space points. By smooth interpolation, we may assume that $\partial_{t} s_{t}(x)=\partial_{x} s_{t}(x)$

\section{Generation of Price Forward Curves: theoretical background}

In our empirical analysis we employed a unique data set of hourly price forward curves $(\mathrm{HPFC}) G_{t}\left(x_{1}\right), \ldots, G_{t}\left(x_{N}\right)$ generated each day between $01 / 01 / 2009$ and $15 / 07 / 2015$ based on the latest information from the observed futures prices for the German electricity Phelix price index. We choose the distance between the maturity points to be $\Delta x=1$ day, but will also in some instances consider longer maturity time steps in our 
analysis. However, unless otherwise explicated, $\Delta x=1$ day is the choice. In this section we describe how these curves were produced from market prices.

For the derivation of the HPFCs we follow the approach introduced by Fleten and Lemming (2003). At any given time the observed term structure at EEX is based only on a limited number of traded futures/forward products. Hence, a theoretical hourly price curve, representing forwards for individual hours, is very useful but must be constructed using additional information. We model the hourly price curve by combining the information contained in the observed bid and ask prices with information about the shape of the seasonal variation.

Recall that $G_{t}(x)$ is the price of the forward contract with time to maturity $x$, where time is measured in hours, and let $F_{t}\left(T_{1}, T_{2}\right)$ be the settlement price at time $t$ of a forward contract with delivery in the interval $\left[T_{1}, T_{2}\right]$. The forward prices of the derived curve should match the observed settlement price of the traded futures product for the corresponding delivery period, that is:

$$
\frac{1}{\sum_{\tau=T_{1}}^{T_{2}} \exp (-r \tau / a)} \sum_{\tau=T_{1}}^{T_{2}} \exp (-r \tau / a) G_{t}(\tau-t)=F_{t}\left(T_{1}, T_{2}\right)
$$

where $r$ is the continuously compounded rate for discounting per annum and $a$ is the number of hours per year. A realistic price forward curve should capture information about the hourly seasonality pattern of electricity prices. For the derivation of the seasonality shape of electricity prices we follow Paraschiv (2013) and Paraschiv, Fleten, and Schürle (2015). Basically we fit the HPFC to the seasonality shape by minimizing

$$
\min \left[\sum_{x=1}^{N}\left(G_{t}(x)-s_{t}(x)\right)^{2}\right]
$$

subject to constraints of the type given in equation $(8)$ for all observed instruments, where 
$s_{t}$ is the hourly seasonality curve (we refer to Fleten and Lemming (2003) for details) ${ }^{4}$

We offer a detailed description of the methodology used to derive the seasonality shape for the Phelix electricity prices in the internet appendix A. To keep the optimization problem feasible, we follow the standard procedure (see Benth, Koekebakker, and Ollmar (2007)) to remove overlapping contracts as well as contracts with delivery periods which are completely overlapped by other contracts with shorter delivery periods. From noarbitrage relationships (see Benth, Šaltytė Benth, and Koekebakker (2008, Eq. (6.6) on p. 165)), there is no information loss in removing a futures contract with delivery period that is overlapped by one or more other futures contracts.

An alternative approach to extract power forward curves from a discrete set of traded contract using spline interpolation is suggested by Benth, Koekebakker, and Ollmar (2007). Recently, Caldana, Fusai, and Roncoroni (2016) proposed a method combining non-parametric filtering with convex interpolation.

\section{Empirical analysis}

The original input to our analysis are 2'386 hourly price forward curves for PHELIX, the German electricity index, generated each day between 01/01/2009 and 15/07/2015, for a horizon of 5 years. The curves have been provided by the Institute of Operations Research and Computational Finance, University of St. Gallen and have been generated consistently based on the approach described in section 3. In a first step, we eliminated the deterministic component of the hourly price forward curves, as shown in Equation (2). To keep the analysis tractable, we chose to work with daily, instead of hourly curves. Thus, the stochastic component of each hourly price forward curve, $f_{t}(x)$, has been filtered

\footnotetext{
${ }^{4}$ In the original model, Fleten and Lemming (2003) applied, for daily time steps, a smoothing factor to prevent large jumps in the forward curve. However, in the case of hourly price forward curves, Bloechlinger (2008) (p. 154) concludes that the higher the relative weight of the smoothing term, the more the hourly structure disappears. We want that our HPFC reflects the hourly pattern of electricity prices and therefore in this study we have set the smoothing term in Fleten and Lemming (2003) to 0.
} 


\footnotetext{
${ }^{5}$ For the generation of PFCs on horizons longer than 2 years, only yearly futures are still observed, so the information about the market expectation becomes more general. We therefore decided to keep the analysis compact and analyse 2 years long truncated curves.
}

\subsection{Analysis of the risk premium}

In the case of storable commodities, arbitrage-based arguments imply that the forward price is equal to the spot price times discount factors involving the risk-free interest rate, storage costs and the convenience yield (see Geman (2005)). However, electricity is nonstorable, so this link does not exist here. Therefore, it can be expected that forward prices are formed as the sum of the expected spot price plus a risk premium that is paid by riskaverse market participants for the elimination of price risk. We estimated Equation (6) for each time-series $Z_{t}(x)$ and $f_{t}(x), t \in\{1, \ldots, T\}$ of each point $x \in\left\{x_{1}, \ldots, x_{N}\right\}$. For taking $\Delta t=1$ day and $\Delta x=1$ day, the estimated risk premia will be a $(1 \times(N-1))$ vector. Estimation results are shown in Figure 3 .

We observe that the risk premia take values between a minimum of -0.086 and maximum 0.017. They oscillate around zero and have a higher volatility over the first three quarters of the year along the curve, so for shorter time to maturities. However, 
on the medium/long-run the risk premia are predominantly negative and their volatility seems to stabilize for the second year.

The finding that the short-term risk premia oscillate around zero is consistent with the findings in the literature. For example Pietz $(2009)$ found that the risk premium may be positive or negative, depending on the average risk aversion in the market. It may vary in magnitude and sign throughout the day and between seasons. Furthermore, Paraschiv, Fleten, and Schürle (2015) found that short-term risk premia are positive during the week and decrease or become negative for the weekend. The disentangled pattern of risk premia between seasons, working/weekend days cannot be investigated here directly, though, since we used for the estimation a time-series of each point along one curve, making use of all generated PFCs used as input. We are in fact interested to examine the evolution of risk premia with increasing time to maturity. In the longrun, the negative risk premia confirm previous findings in the literature (see e.g. Burger, Graeber, and Schindlmayr (2007)): producers accept lower futures prices, as they need to make sure that their investment costs are covered.

\subsection{Analysis of term structure volatility}

In Figure 4 we plot the term structure volatility $\sigma(x)$, for $x \in\left\{x_{1}, \ldots, x_{N}\right\}$, as defined in Equation (7). Overall we observe that the volatility decreases with increasing time to maturity. In particular, it decays faster for shorter time to maturity and it shows a bump around the maturity of 1 month. Around the second (front) quarter the volatility starts increasing again, showing a second bump around the third quarter. The reason is that for time to maturities longer than one month, in most of the cases weekly futures are not available anymore, so the next shortest maturity available in the market is the front month future. That means: if market participants are interested in one sub-delivery period within the second month, there are no weekly futures available to properly price their contracts, but the only available information is from the front month futures price. 


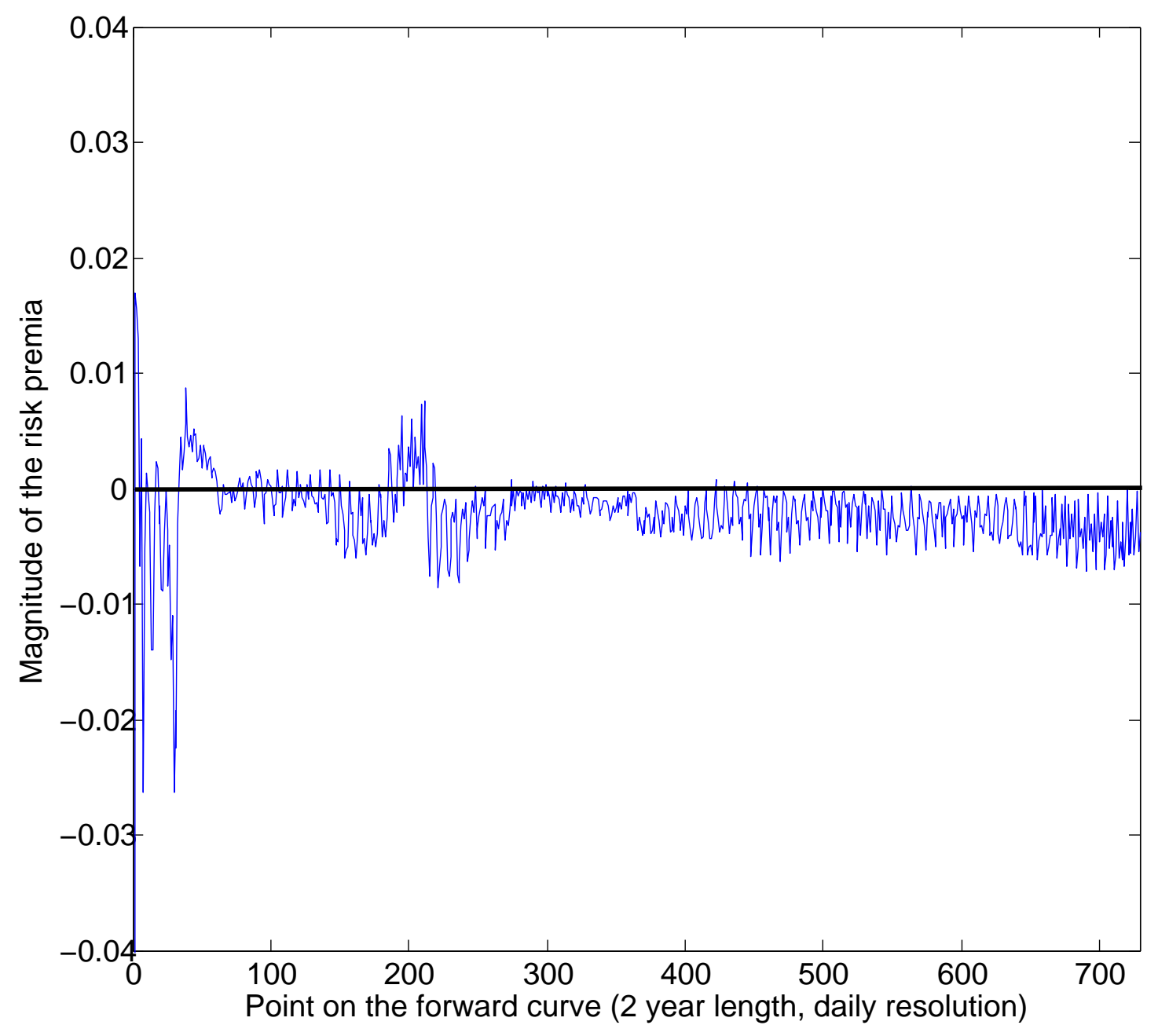

Figure 3: Risk premia along one curve (2-year length, daily resolution) 
It is known that the volume of trades for this front month future increases, thus inducing a higher volatility of the corresponding forward prices. In Figure 5 we observe that indeed, the front month future has the highest and the most volatile volume of trades over the investigated time period, compared to the other monthly traded contracts. A similar effect is around the front quarter, when monthly futures are not observed anymore, but the information about the level of the (expected) price is given by the corresponding quarterly future contract. In consequence, the volume of trades for the front quarterly future and for the 2nd available quarterly future increases, these being the most traded products in the market, as shown in Figure 6. This explains the increase in the volatility during the front quarter segment of the forward curve and the second bump.

The jigsaw pattern of the volatility curve reflects the weekend effect: the volatility of forwards is lower during weekend versus working days. A similar pattern is observed in the spot price evolution, as shown in Paraschiv, Fleten, and Schürle (2015).

\subsection{Statistical properties of the noise time series}

The analysis of the noise time-series $\tilde{\epsilon}_{t}$ (see Equation (7)) is twofold: First, we examine the statistical properties of individual time series $\tilde{\epsilon}_{t}\left(x_{i}\right)$ and in particular we check for stationarity, autocorrelation and ARCH/GARCH effects. Secondly, we examine patterns in the correlation matrix with respect to the time/maturity dimensions. Thus, we are interested in the correlations between $\tilde{\epsilon}_{t}\left(x_{i}\right)$ and $\tilde{\epsilon}_{t}\left(x_{j}\right)$, for $i, j \in\{1, \ldots, N\}, t=1, \ldots, T$ to examine the effect of the time to maturity on the joint dynamics between the noise components. Furthermore, we are interested in the correlations between noise curves, with respect to the points in time where these have been generated: correlations between $\tilde{\epsilon}_{m}\left(x_{1}\right), \ldots, \tilde{\epsilon}_{m}\left(x_{N}\right)$ and $\tilde{\epsilon}_{n}\left(x_{1}\right), \ldots, \tilde{\epsilon}_{n}\left(x_{N}\right)$, for $m, n \in\{1, \ldots, T\}$. The analysis is performed initially for taking $\Delta x=1 d a y$ and $\Delta t=1 d a y$, as defined in Equation (5).

We are further interested to see whether the statistical properties of the noise as well as the correlations between its components change, if we vary the maturity step 


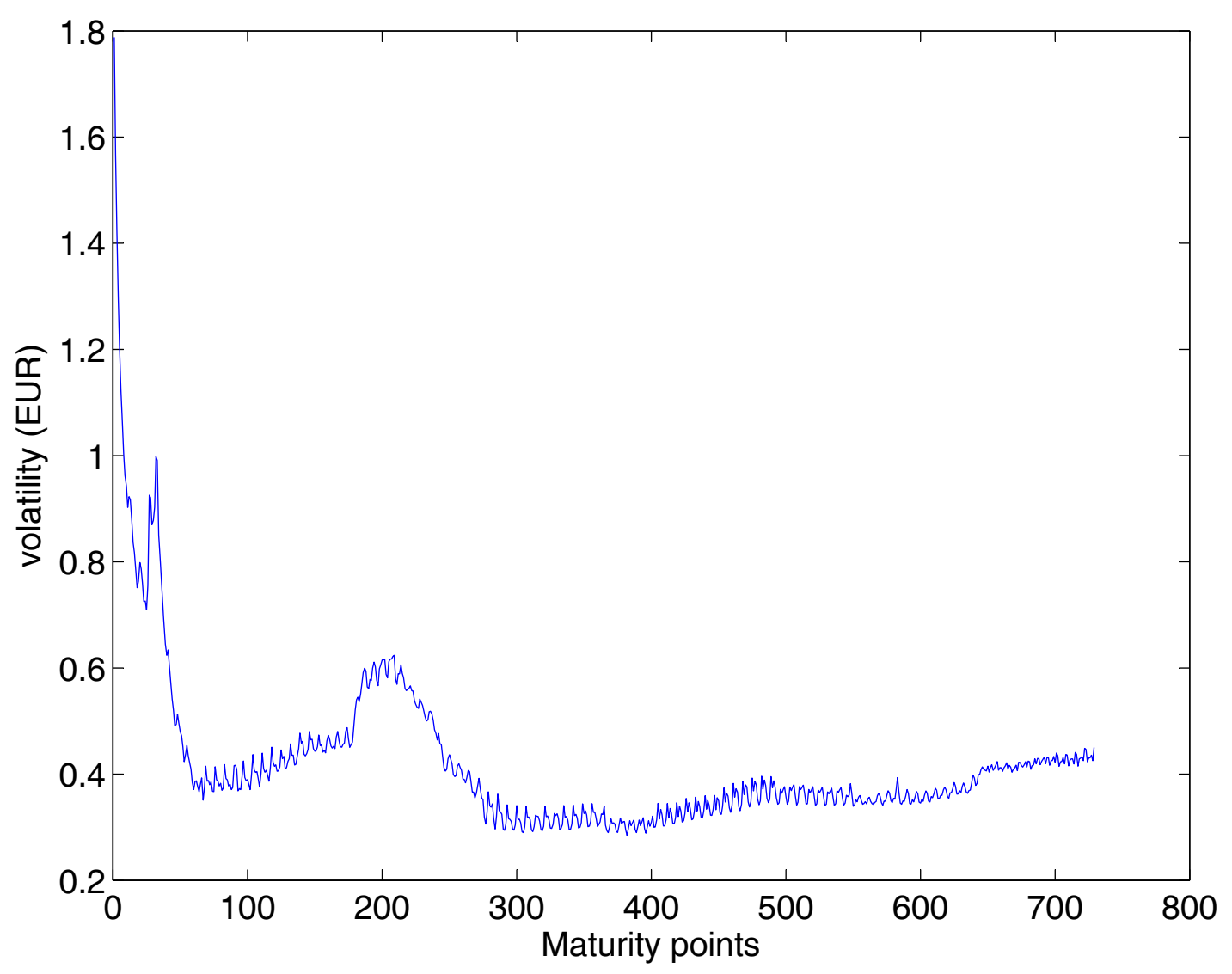

Figure 4: The empirical volatility term structure 


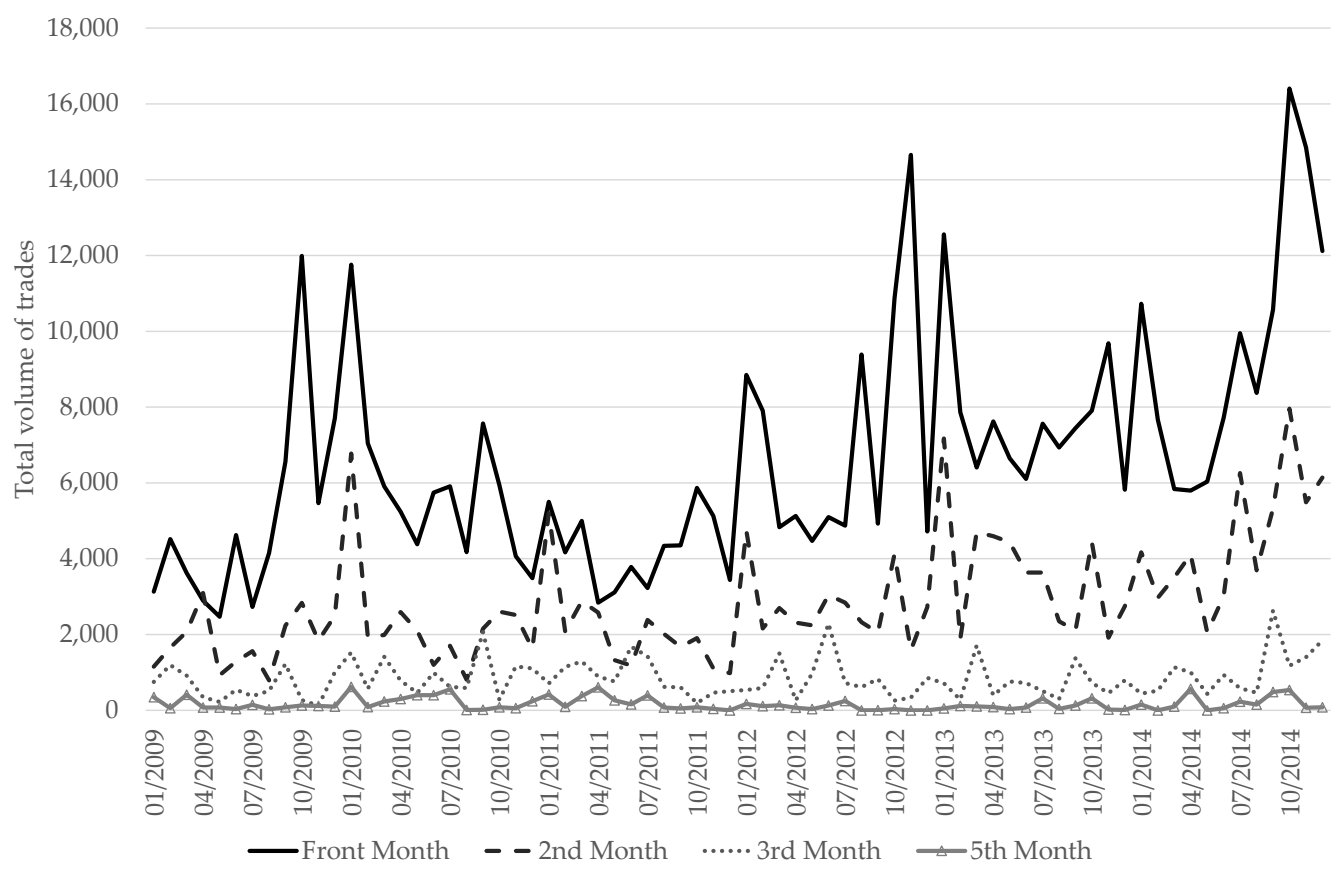

Figure 5: The sum of traded contracts for the monthly futures at EPEX (own calculations, source of data: ems.eex.com).

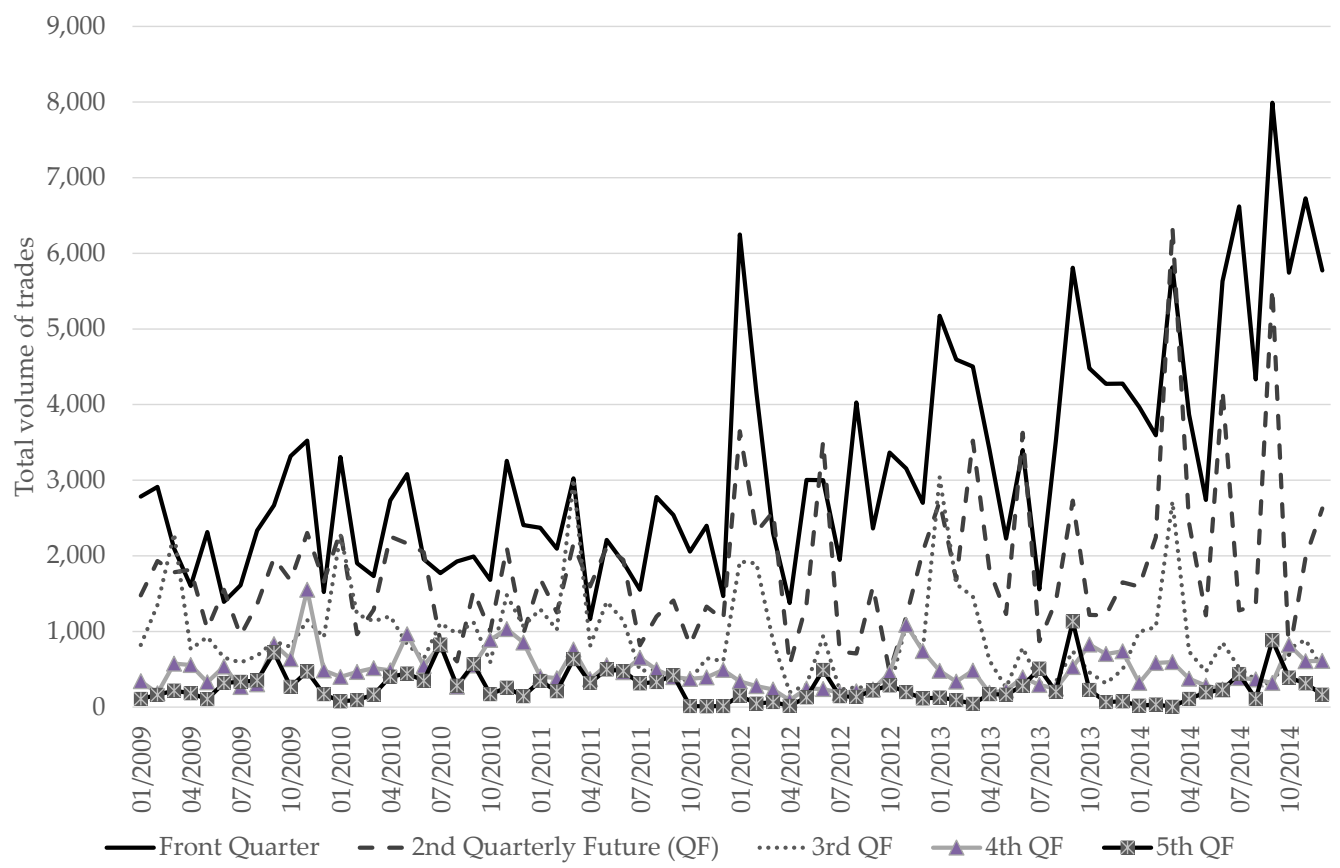

Figure 6: The sum of traded contracts for the quarterly futures at EPEX (own calculations, source of data: ems.eex.com). 
$\Delta x$ in Equation (5) (and implicitly $\Delta t$ ). When changing $\Delta x$, we are also changing the numerical differentiation in Equation (5) with respect to $x$, and in particular the range (in the maturity direction) for when information is accounted for. Various maturity steps may lead to slightly different properties of the noise, given the stepwise pattern of the deaseasonalized price forward curves $f_{t}(x)$, as shown in Figures 1 and 2 in the internet appendix B. The stepwise pattern comes from the different level of futures prices of different maturities taken as input for the generation of price forward curves. Futures have different delivery periods, weekly, monthly, quarterly, yearly, and at each point when a new future is observed, the level in the generated price forward curve changes (recall Figure 1). As the choice of $\Delta x$ dictates when information from observed futures contracts is accounted for, it will impact the generated price forward curve. This is taken over in the stochastic component $f_{t}(x)$. Furthermore, within one week, we observe the weekend effect: the price level is different between working/weekend days. All these cause sparse matrices in the noise, given the many values of "zero" obtained after differentiating.

To assess the impact of stepwise changes in the stochastic component of price forward curves $f_{t}(x)$, we replicated the analysis for one additional case study: We further investigated the effect of a change between consecutive weekly futures prices by taking $\Delta x=7$ days. This choice of maturity step further affects the impact from monthly and quarterly products on the level of the generated curve.

\subsubsection{Stationarity, Autocorrelation, ARCH/GARCH effects}

The stationarity, autocorrelation pattern and $\mathrm{ARCH} / \mathrm{GARCH}$ effects are computed for each case study of $\Delta x / \Delta t$, namely 1 day and 7 days shifts in maturity (and time). To reduce the complexity, we compute these statistics for time series of equidistant points along the curve's length: $\tilde{\epsilon}_{t}\left(x_{k}\right)$, where $k \in\{1, \ldots, N\}$. In choosing $k$ we increment over 90 days (approximately one quarter) along one noise curve. To test for stationarity, we applied the Augmented Dickey-Fuller (ADF) and Phillips-Perron tests for a unit root in 
each univariate time series $\tilde{\epsilon}_{t}\left(x_{k}\right)$. Results are confirmed when applying the KwiatkowskiPhillips-Schmidt-Shin test statistic for stationarity (with intercept, no trend). Results are available in Tables 1 and 2 for the case studies $\Delta x=1$ day and $\Delta x=7$ days, respectively. For $h=0$, we fail to reject the null that series are stationary. Unit root test results are shown in detail in Table 3. Thus, all statistical tests conclude that time series $\tilde{\epsilon}_{t}\left(x_{k}\right)$ are stationary.

We further tested the hypothesis that the $\tilde{\epsilon}_{t}\left(x_{k}\right)$ series are autocorrelated. Autocorrelation test results are shown in Tables 1 and 2 . We replicated the test for the level of the noise time series and for their squared values (columns 2 and 3 , respectively). $h 1=0$ indicates that there is not enough evidence to suggest that noise time series are autocorrelated. In Figures 7 and 8 we display the autocorrelation function for series $\tilde{\epsilon}_{t}\left(x_{k}\right)$ for $k \in\{90,180,270,360\}$, for the level and squared residuals, respectively. In the first case, the pattern of the autocorrelation function for the level of residuals shows a typical white noise pattern. Still, as expected, the autocorrelation function shows a slight decaying pattern in the second case (Figure 8), where we look at the squared residuals. The decaying pattern becomes more obvious when we move to the case study two, where the change in maturity (and time) is set to 7 days, as shown in Figure 9. This is not surprising, since an increment of maturity points and time of 7 days leads to less zero increments in the noise time series overall, which allows a more visible pattern of autocorrelation. Results of the autocorrelation test conclude our findings from the visual inspection: if in the basic case study of $\Delta x=1$ day we did not find evidence for autocorrelation in all time series of the noise (Table 1, second and third columns), there is clear evidence for autocorrelation in all series with increasing maturity step $\Delta x=7$ days.

We further tested the hypothesis that there are significant $\mathrm{ARCH}$ effects in the $\tilde{\epsilon}_{t}\left(x_{k}\right)$ series by employing the Ljung-Box Q-Test. Results are shown in the last columns of Tables 11 and 2, $h 2=1$ indicates that there are significant ARCH effects in the noise time-series. Independent of the maturity/time step chosen, time series are characterized 
by $\mathrm{ARCH}$ effects, and thus by a volatility clustering pattern. In Equation (7) we filter the volatility out of the marginal noise $\epsilon_{t}(x)$. However, the volatility is not time-varying in our model, which explains that there is evidence for remaining stochastic volatility (conditional heteroscedasticity) in the standardized residuals $\tilde{\epsilon}_{t}(x)$. We tested for a unit root in the unobserved volatility process by testing for a unit root in the log of the squared time series. Standard unit root tests (ADF, PP, KPSS) are known to suffer from extreme size distortions in the presence of negative mean average (MA) roots which are expected to occur, given the identified ARCH/GARCH results (see Wright (1999)). We therefore apply the methodology in Perron and Ng (1996) who have proposed modified unit root tests which are robust to large negative MA roots. As shown in Table 4, NG-Perron test statistics show evidence for a unit root in the volatility process.

In the light of the identified $\mathrm{ARCH} / \mathrm{GARCH}$ effects in the marginals $\tilde{\epsilon}_{t}\left(x_{k}\right)$, we inspect their tail behavior by plotting the kernel smoothed empirical densities versus normal distribution for series $k \in\{1,90,180,270\}$, as shown in Figure 11. We observe the strong leptokurtic pattern of heavy tailed marginals.

Overall we conclude that the model residuals are coloured noise, with heavy tails (leptokurtic distribution) and with a tendency for conditional volatility.

\subsubsection{Spatial Correlation}

In the autocorrelation functions examined above, we show that there are temporal correlations between forward curves produced at different points in time. In addition, we are interested in the spatial correlation structure between $\tilde{\epsilon}_{t}\left(x_{i}\right)$ and $\tilde{\epsilon}_{t}\left(x_{j}\right)$, for $i, j \in 1, \ldots, N$, to examine how noise correlations change with increasing distance between the maturity points along one curve. In Figure 10 we observe that correlations oscillate between positive and negative, which is expected, given the nature of the (coloured) noise time series (stationary, oscillating around 0). As expected, spatial correlations between maturity points of up to 1 month (about 30 day) decay fast with increasing distance between 


\begin{tabular}{lrrrr}
\hline \hline$\tilde{\epsilon}_{t}\left(x_{k}\right)$ & Stationarity & Autocorrelation $\tilde{\epsilon}_{t}\left(x_{k}\right)$ & Autocorrelation $\tilde{\epsilon}_{t}\left(x_{k}\right)^{2}$ & ARCH/GARCH \\
\hline & $\mathrm{h}$ & $\mathrm{h} 1$ & $\mathrm{~h} 1$ & $\mathrm{~h} 2$ \\
\hline Q0 & 0 & 1 & 1 & 1 \\
Q1 & 0 & 0 & 0 & 0 \\
Q2 & 0 & 1 & 1 & 1 \\
Q3 & 0 & 0 & 1 & 1 \\
Q4 & 0 & 1 & 0 & 0 \\
Q5 & 0 & 1 & 1 & 1 \\
Q6 & 0 & 1 & 1 & 1 \\
Q7 & 0 & 1 & 1 & 1 \\
\hline \hline
\end{tabular}

Table 1: The time series are selected by quarterly increments (90 days) along the maturity points on one noise curve. Hypotheses tests results, case study 1: $\Delta x=1$ day. In column 'Stationarity', if $h=0$ we fail to reject the null that series are stationary. For 'Autocorrelation', $h 1=0$ indicates that there is not enough evidence to suggest that noise time series are autocorrelated. In the last column, $h 2=1$ indicates that there are significant ARCH effects in the noise time-series.

\begin{tabular}{lrrrr}
\hline \hline$\tilde{\epsilon}_{t}\left(x_{k}\right)$ & Stationarity & Autocorrelation $\tilde{\epsilon}_{t}\left(x_{k}\right)$ & Autocorrelation $\tilde{\epsilon}_{t}\left(x_{k}\right)^{2}$ & ARCH/GARCH \\
\hline & $\mathrm{h}$ & $\mathrm{h} 1$ & $\mathrm{~h} 1$ & $\mathrm{~h} 2$ \\
\hline Q0 & 0 & 1 & 1 & 1 \\
Q1 & 0 & 1 & 1 & 1 \\
Q2 & 0 & 1 & 1 & 1 \\
Q3 & 0 & 1 & 1 & 1 \\
Q4 & 0 & 1 & 1 & 1 \\
Q5 & 0 & 1 & 1 & 1 \\
Q6 & 0 & 1 & 1 & 1 \\
Q7 & 0 & 1 & 1 & 1 \\
\hline \hline
\end{tabular}

Table 2: The time series are selected by quarterly increments (90 days) along the maturity points on one noise curve. Hypotheses tests results, case study 2: $\Delta x=7$ days. In column 'Stationarity', if $h=0$ we fail to reject the null that series are stationary. For 'Autocorrelation', $h 1=0$ indicates that there is not enough evidence to suggest that noise time series are autocorrelated. In the last column, $h 2=1$ indicates that there are significant ARCH effects in the noise time-series. 

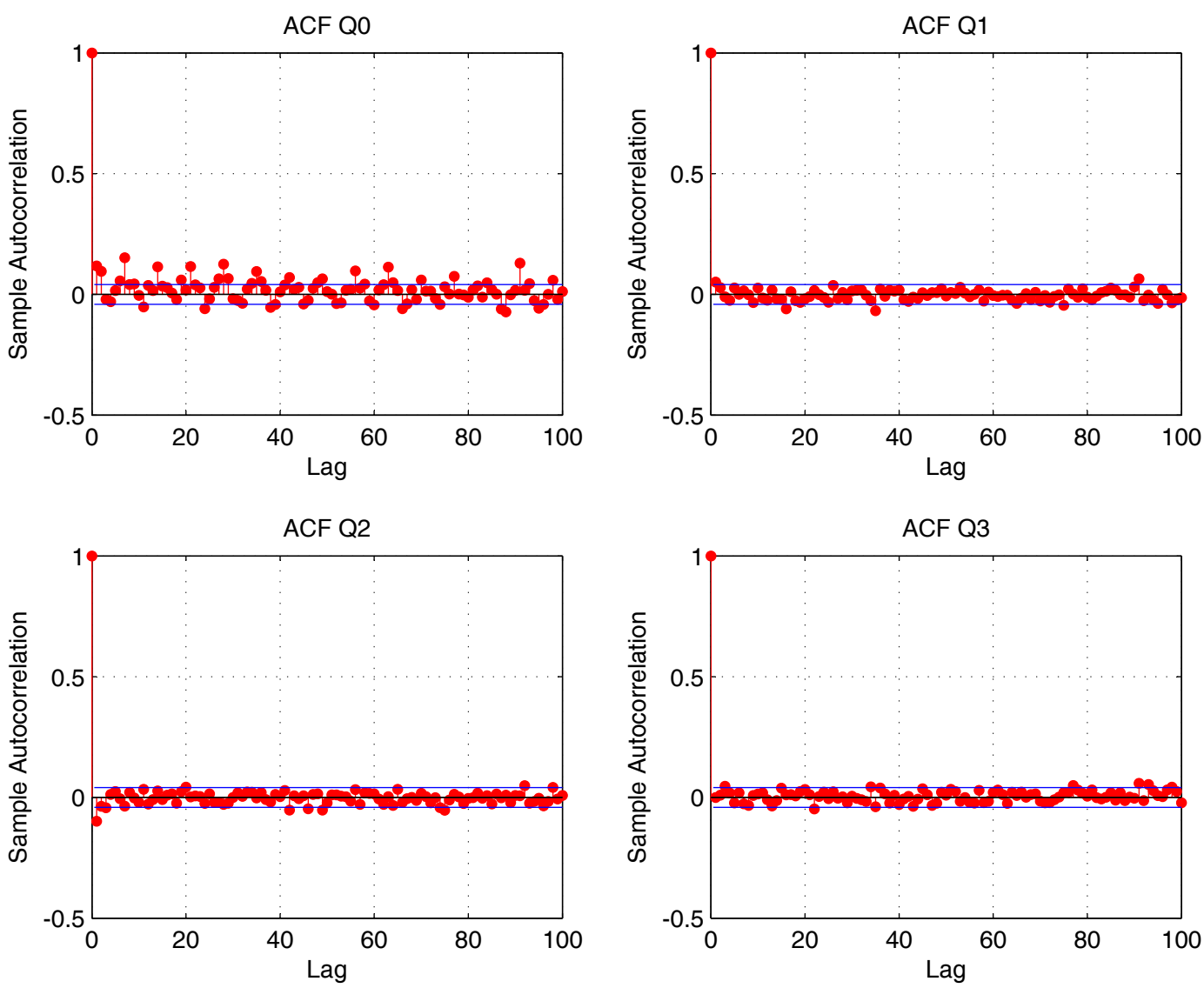

Figure 7: Autocorrelation function in the level of the noise time series $\tilde{\epsilon}_{t}\left(x_{k}\right)$, by taking $k \in\{1,90,180,270\}$, case study 1: $\Delta x=1$ day. 

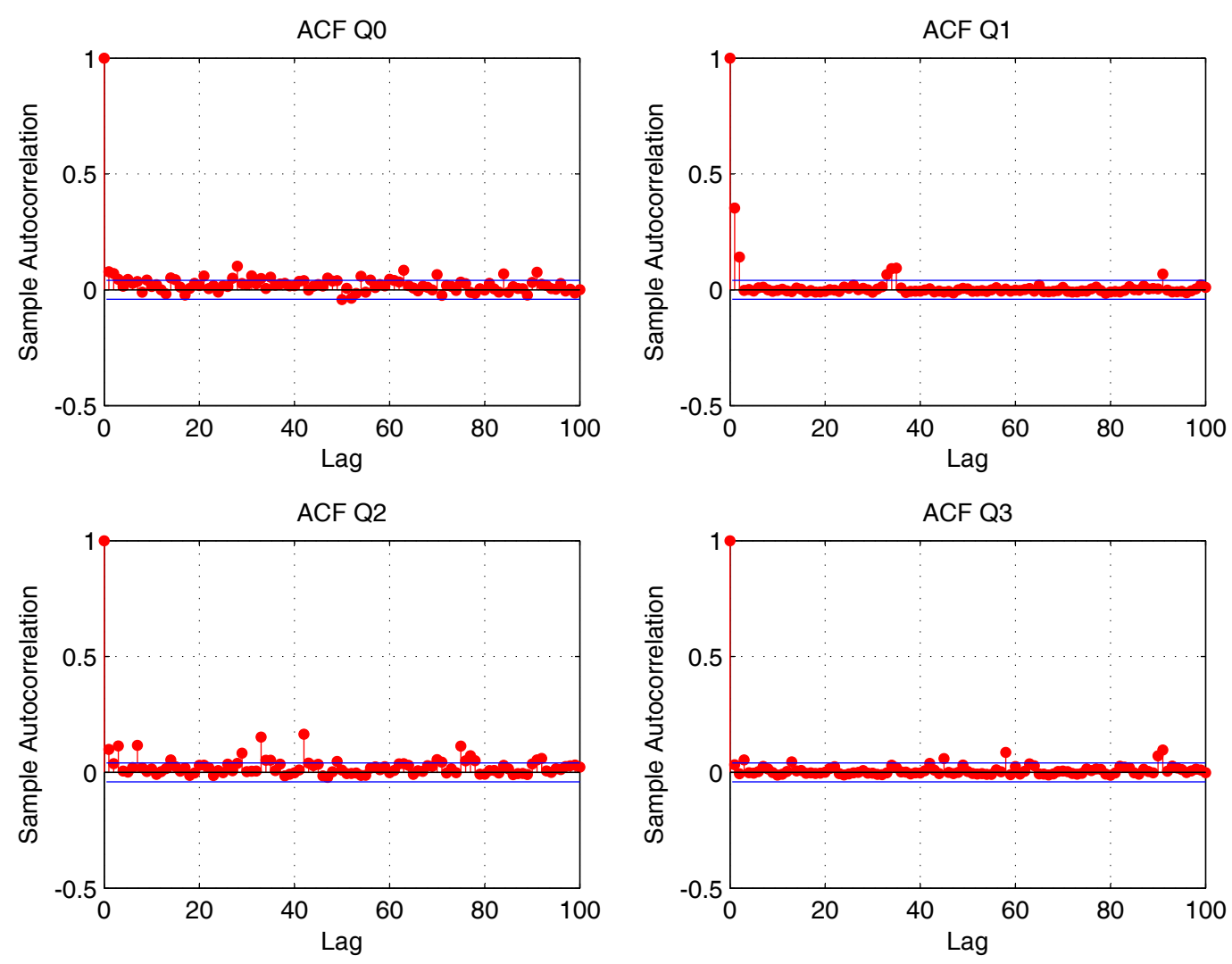

Figure 8: Autocorrelation function in the squared time series of the noise $\tilde{\epsilon}_{t}\left(x_{k}\right)^{2}$, by taking $k \in\{1,90,180,270\}$, case study 1: $\Delta x=1$ day. 

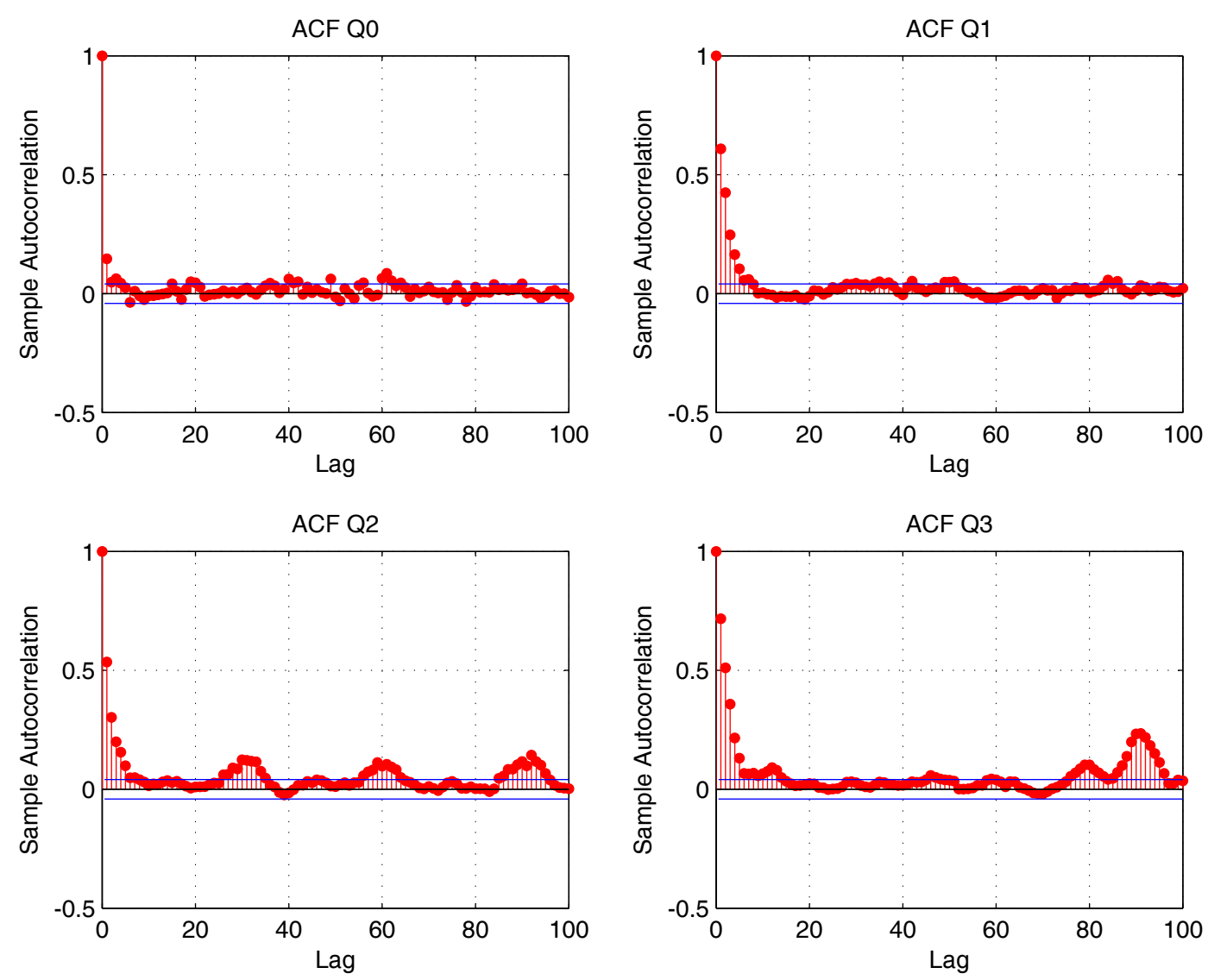

Figure 9: Autocorrelation function in the squared time series of the noise $\tilde{\epsilon}_{t}\left(x_{k}\right)^{2}$, by taking $k \in\{1,90,180,270\}$, case study $2: \Delta x=7$ days. 


\begin{tabular}{l|r|r|r|r|r}
\hline \hline Test & Null hypothesis & Q0 & Q1 & Q2 & Q3 \\
\hline ADF test & Unit root & $-4.476^{*}$ & $-4.701^{*}$ & $-3.504^{*}$ & $-3.600^{*}$ \\
PP test & Unit root & $-52.550^{*}$ & $-51.755^{*}$ & $-52.623^{*}$ & $-52.720^{*}$ \\
KPSS test & Stationarity & 0.564 & 0.399 & 0.329 & 0.367 \\
\hline \hline
\end{tabular}

Table 3: Unit root test results for series $\tilde{\epsilon}_{t}\left(x_{k}\right)$ for quarterly increments in $k \in 1,90,180,270$. Note: One star denotes significance at the 1\% level. ADF refers to Augmented Dickey-Fuller test, PP to the Philips-Peron test and KPSS to the Kwiatkowski-Phillips-Schmidt-Shin test. The lag structure of the ADF test is selected automatically on the basis of the Bayesian Information Criterion (BIC). For PP and KPSS tests the bandwidth parameter is selected according to the approach suggested by Newey and West (1994).

\begin{tabular}{l|r|r|r|r}
\hline \hline NG-Perron test & Q0 & Q1 & Q2 & Q3 \\
\hline MZa & -2.457 & -1.719 & -1.901 & -1.382 \\
$\mathrm{MZt}$ & -0.967 & -0.837 & -0.891 & -0.731 \\
\hline \hline
\end{tabular}

Table 4: NG-Perron unit root test results for series $\log \left(\tilde{\epsilon}_{t}\left(x_{k}\right)^{2}\right)$ for quarterly increments in $k \in 1,90,180,270$. Note: We test the null hypothesis: series has a unit root. One star denotes significance at the $1 \%$ level. MZa and MZt are the three modified Z-test statistics of Perron and $\mathrm{Ng}$ (1996). The lag length of the NG-Perron test is selected automatically on the basis of the Spectral GLS-detrended AR based on Schwarz Information Criteria (SIC).

them. This reflects the higher interest of market participants for maturing contracts. The correlations between maturity points situated at distances longer than 30 days are very low, oscillating around zero. However, correlations between 1 year distant maturity points slightly increase. This shows that the stochastic component of forward prices is driven by common factors at the same time of the year, which is reflected in a higher correlation between yearly futures products.

\section{Modeling approach and estimation of the noise}

Given the heavy tails of marginals identified in Figure 11, we model the noise marginals $\tilde{\epsilon}_{t}(x)$ by a Normal Inverse Gaussian distribution (NIG). The NIG distribution is a special case of the Generalized Hyperbolic Distribution for $\lambda=-1 / 2$ and its density reads (see 


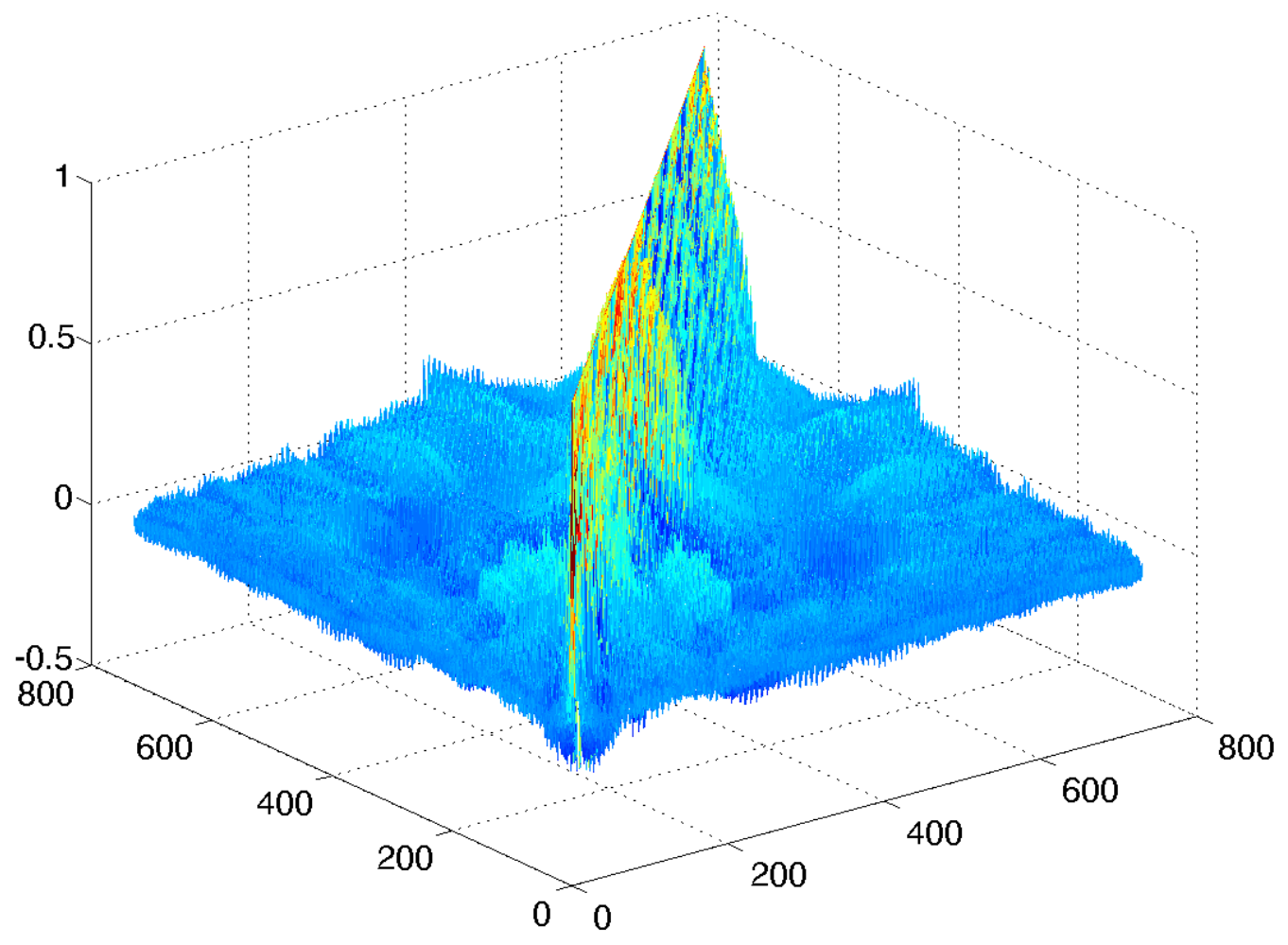

Figure 10: Correlation matrix with respect to different maturity points 


\begin{tabular}{lrrrr}
\hline \hline Parameter & $\mathrm{Q} 0$ & $\mathrm{Q} 1$ & $\mathrm{Q} 2$ & $\mathrm{Q} 3$ \\
\hline$\delta$ & 1.115 & 0.252 & 0.193 & 0.226 \\
& $(0.102)$ & $(0.007)$ & $(0.005)$ & $(0.006)$ \\
$\alpha$ & 1.083 & 0.188 & 0.116 & 0.195 \\
& $(0.152)$ & $(0.046)$ & $(0.052)$ & $(0.037)$ \\
$\beta$ & 0.111 & -0.012 & 0.000 & -0.001 \\
& $(0.054)$ & $(0.024)$ & $(0.022)$ & $(0.021)$ \\
$\mu$ & -0.011 & 0.000 & -0.004 & -0.002 \\
& $(0.049)$ & $(0.004)$ & $(0.007)$ & $(0.007)$ \\
\hline \hline
\end{tabular}

Table 5: Maximum likelihood estimates of NIG to $\tilde{\epsilon}_{t}\left(x_{k}\right)$ by taking $k \in\{1,90,180,270\}$ for $\mathrm{Q} 0, \ldots, \mathrm{Q} 3$, respectively.

Standard errors are shown in parentheses.

Benth, Šaltytė Benth, and Koekebakker (2008)):

$$
f_{N I G}(x)=\frac{\alpha}{\pi} \exp \left(\delta \sqrt{\alpha^{2}-\beta^{2}}+\beta(x-\mu)\right) \frac{K_{1}\left(\alpha \delta \sqrt{1+\left(\frac{x-\mu}{\delta}\right)^{2}}\right)}{\sqrt{1+\left(\frac{x-\mu}{\delta}\right)^{2}}}
$$

We have firstly fitted a NIG by moment estimators. We observed that the fitted density performs visibly better than a normal distribution in explaining the leptokurtic pattern of time series. In a second step, we estimated NIG by maximum likelihood (ML). The mathematical formulation of the likelihood function and related gradients as input to the numerical optimization procedure are given in the internet appendix C.

The ML estimates improved further the fit of the NIG density. In Table 5 we show the ML estimates for the NIG distribution fitted to $\tilde{\epsilon}_{t}\left(x_{k}\right)$ by taking $k \in\{1,90,180,270\}$. In Figure 11 we show the kernel density estimates versus normal and the two versions of the NIG estimation. We confirm a realistic performance of the NIG distribution in explaining the heavy tail behavior of noise marginals. 

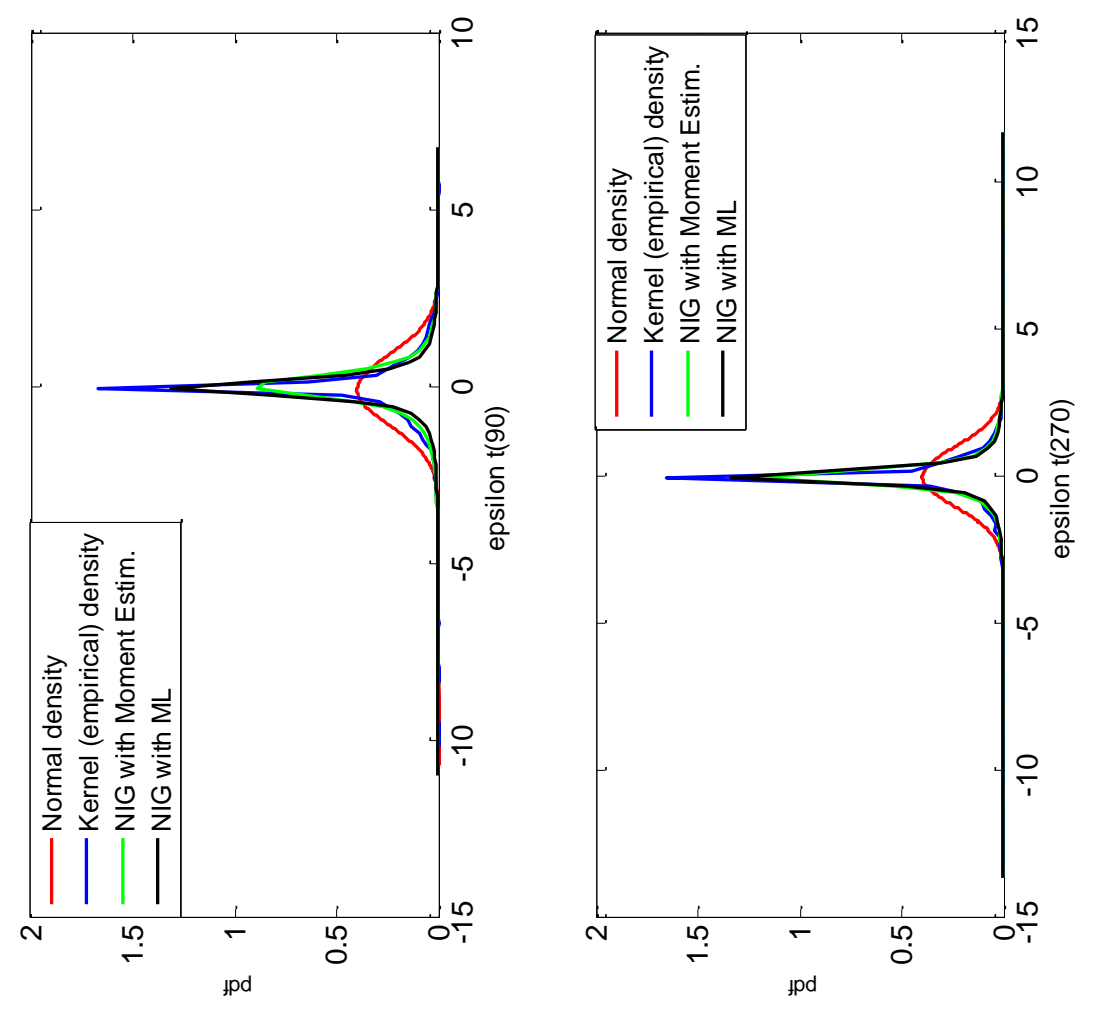

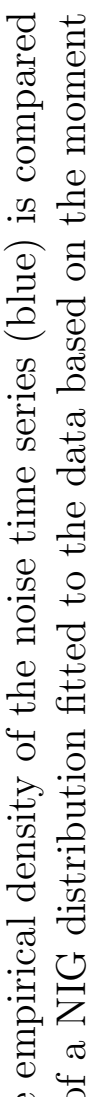

E.

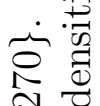
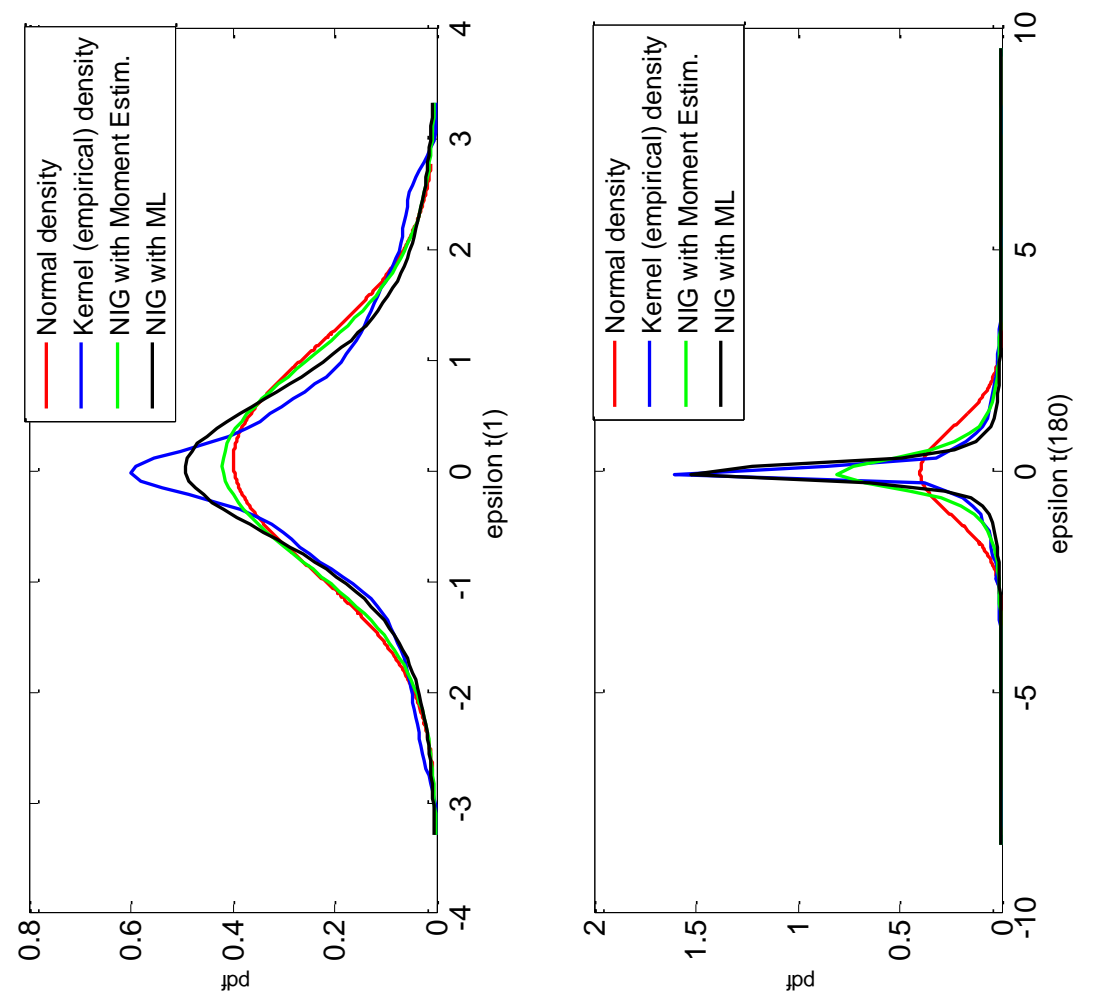

$\infty \underset{1}{\infty}$

ᄋㅇำ

记

4 ซ의

$\leqslant \underset{0}{0}$

氙

उ鸷

है

心:

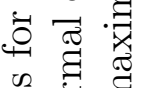

号苛

을

空㟧

㠻.

$\ddot{\exists} \underset{0}{0}$

○

斻 


\section{Revisiting the spatio-temporal model of forward prices}

In our empirical analysis of EPEX electricity forward prices, we have made use of a time series discretization of the deseasonalized term structure dynamics $f_{t}(x)$ defined in (3). We have estimated the parameter of the market price of risk $\theta(x)$, and have analysed empirically the noise residual $d W_{t}(x)$ expressed as $\epsilon_{t}(x)=\sigma(x) \widetilde{\epsilon}_{t}(x)$ in a discrete form in (7). The purpose of this Section is to recover an infinite dimensional model for $W_{t}(x)$ based on our findings.

To this end, we recall that $\mathcal{H}$ is a separable Hilbert space of real-valued functions on $\mathbb{R}_{+}$, where $W_{t}$ is a martingale process. As suggested by the notation, a first model could simply be to assume that $W$ is a $\mathcal{H}$-valued Wiener process. However, this would mean that we expect $t \mapsto W_{t}(x)$ to be a Gaussian process for each $x \geq 0$, which is at stake with our empirical findings showing clear non-Gaussian (or, coloured noise) residuals. After explaining the Samuelson effect, the residuals could be modelled nicely by a NIG distribution.

In stochastic modelling of financial price dynamics, it is common to scale the random variations by a volatility factor. We follow this idea, and propose a model of $W$ of the form

$$
W_{t}=\int_{0}^{t} \Sigma_{s} d L_{s}
$$

where $s \mapsto \Sigma_{s}$ is an $L(\mathcal{U}, \mathcal{H})$-valued predictable process and $L$ is a $\mathcal{U}$-valued Lévy process with zero mean and finite variance. We refer to (Peszat and Zabczyk, 2007, Sect. 8.6) for conditions to make the stochastic integral well-defined. As a first case, we can choose $\Sigma_{s} \equiv \Psi$ time-independent, being an operator mapping elements of the separable Hilbert space $\mathcal{U}$ into $\mathcal{H}$. An increment in $W_{t}$ can be approximated (based on the definition of the 
stochastic integral, see (Peszat and Zabczyk, 2007, Ch. 8)) as

$$
W_{t+\Delta t}-W_{t} \approx \Psi\left(L_{t+\Delta t}-L_{t}\right)
$$

Choose now $\mathcal{U}=L^{2}(\mathbb{R})$, the space of square integrable functions on the real line equipped with the Lebesgue measure, and assume $\Psi$ is an integral operator on $L^{2}(\mathbb{R})$, i.e., for $g \in L^{2}(\mathbb{R})$, the mapping

$$
\mathbb{R}_{+} \ni x \mapsto \Psi(g)(x)=\int_{\mathbb{R}} \tilde{\sigma}(x, y) g(y) d y
$$

defines an element in $\mathcal{H}$. Furthermore, if $\operatorname{supp} \widetilde{\sigma}(x, \cdot)$ is concentrated in a close neighborhood of $x$, we can further make the approximation $\Psi(g)(x) \approx \widetilde{\sigma}(x, x) g(x)$. As a result, we find

$$
W_{t+\Delta t}(x)-W_{t}(x) \approx \widetilde{\sigma}(x, x)\left(L_{t+\Delta t}(x)-L_{t}(x)\right) .
$$

In view of the definition of $\epsilon_{t}(x)$ in $(7)$, we can choose $\sigma(x)=\widetilde{\sigma}(x, x)$ to be the model for the Samuelson effect that we identified and discussed in Subsect. 4.2, and we let $L_{t}$ be a NIG Lévy process with values in $L^{2}(\mathbb{R})$ to model the standardized residuals $\tilde{\varepsilon}_{t}$ (see Benth and Krühner (2015) for a definition of such a process).

Recall from Fig. 10 the spatial correlation structure of $\widetilde{\epsilon}_{t}(x)$. This provides the empirical foundation for defining a covariance functional $\mathcal{Q}$ associated with the Lévy process $L$. In general, we know that for any $g, h \in L^{2}(\mathbb{R})$,

$$
\mathbb{E}\left[\left(L_{t}, g\right)_{2}\left(L_{t}, h\right)_{2}\right]=(\mathcal{Q} g, h)_{2}
$$

${ }_{458}$ where $(\cdot, \cdot)_{2}$ denotes the inner product in $L^{2}(\mathbb{R})($ see $($ Peszat and Zabczyk, 2007, Thm. 4.44)). The covariance functional will be a symmetric, positive definite trace class operator from 
$L^{2}(\mathbb{R})$ into itself. It can be specified as an integral operator on $L^{2}(\mathbb{R})$ by

$$
\mathcal{Q} g(x)=\int_{\mathbb{R}} q(x, y) g(y) d y,
$$

for some suitable "kernel-function" $q$. If $q$ is symmetric, positive definite and continuous function, then it follows from Thm. A.8 in Peszat and Zabczyk (2007) that $\mathcal{Q}$ is a covariance operator of $L$ if we restrict ourselves to $L^{2}(\mathcal{O})$, where $\mathcal{O}$ is a bounded and closed subset of $\mathbb{R}$. Indeed, we can think of $\mathcal{O}$ as the maximal horizon of the market, in terms of relevant times to maturity (recall that we have truncated the forward curves in our empirical study to a horizon of 2 years).

If we assume $g \in L^{2}(\mathbb{R})$ to be close to $\delta_{x}$, the Dirac $\delta$-function, and likewise, $h \in$ $L^{2}(\mathbb{R})$ being close to $\delta_{y},(x, y) \in \mathbb{R}^{2}$, we find approximately

$$
\mathbb{E}\left[L_{t}(x) L_{t}(y)\right]=q(x, y)
$$

This shows that we may interpret the function $q$ as the spatial correlation function of $L$. Unfortunately, the Dirac $\delta$-function $\delta_{x}$ is not an element in $L^{2}(\mathbb{R})$, so we can only obtain the relationship in Equation (16) in an approximative manner. Note that we can approximate $\delta_{x}$ arbitrary well by smooth functions in $L^{2}(\mathbb{R})$, so for practical applications we may use the relation in Equation (16). From the spatial correlation study of $\widetilde{\epsilon}_{t}$, we observe that the correlation is stationary in space in the sense that it only depends on the distance $|x-y|$. Hence, with a slight abuse of notation, we let $q(x, y)=q(|x-y|)$. A simple choice resembling to some degree the fast decaying property in Fig. 10 is $q(|x-y|)=$ $\exp (-\gamma|x-y|)$ for a constant $\gamma>0$. We further note that from Benth and Krühner (2015), it follows that $t \mapsto\left(L_{t}, g\right)_{2}$ is a NIG Lévy process on the real line. If $g \approx \delta_{x}$, then we see that $L_{t}(x)$ for given $x$ is a real-valued NIG Lévy process. With these considerations, we have established a possible model for $W$ which is, at least approximately, consistent with our empirical findings for $\epsilon_{t}$. 
Let us briefly discuss why we suggest to use $\mathcal{U}=L^{2}(\mathbb{R})$ and introduce a rather complex integral operator definition of $\Psi$. As mentioned in Sect. 2, the Hilbert space $\mathcal{H}$ to realize the deseasonalized forward price dynamics $f_{t}$ should be a function space on $\mathbb{R}_{+}$. $L^{2}(\mathbb{R})$ is a space of equivalence classes, and the evaluation operator $\delta_{x}(g)=g(x)$ is not a continuous linear operator on this space. A natural Hilbert space where indeed $\delta_{x}$ is a linear functional (e.g., continuous linear operator from the Hilbert space to $\mathbb{R}$ ) is the so-called Filipovic space. The Filipovic space was introduced and studied in the context of interest rate markets by Filipovic (2001), while Benth and Krühner $(2014,2015)$ have proposed this as a suitable space for energy forward curves. From Benth and Krühner (2014) we have a characterization of the possible covariance operators of Lévy processes in the Filipovic space, which, for example, cannot be stationary in the form suggested for $q$ above. Using $\mathcal{U}=L^{2}(\mathbb{R})$ opens for a much more flexible specification of the covariance operator, which matches nicely the empirical findings on our electricity data. On the other hand, we need to bring the noise $L$ over to the Filipovic space, since we wish to have dynamics of the term structure in a Hilbert space for which we can evaluate the curve at a point $x \geq 0$, that is, $\delta_{x}\left(f_{t}\right)=f_{t}(x)$ makes sense. We recover the actual forward price dynamics $t \mapsto F(t, T)$ in this case by

$$
F(t, T)=f(t, T-t)=\delta_{T-t}\left(f_{t}\right)
$$

We remark that for elements $f$ in the Filipovic space, $x \mapsto f(x)$ will be continuous, and weakly differentiable. To specify $\Psi$ as an integral operator on $L^{2}(\mathbb{R})$, we can bring any element of $L^{2}(\mathbb{R})$ to a smooth function. Indeed, the convolution product of a square integrable function with a smooth function will yield a smooth function (see (Folland, 1984 Prop. 8.10)). This enables us to select "volatility" functions $\widetilde{\sigma}$ ensuring that $W_{t}$ becomes an element of the Filipovic space. Unfortunately, a simple multiplication operator $\Psi(g)(x)=\sigma(x) g(x)$ will not do the job, as this will not be an element of the Filipovic space for general $g \in L^{2}(\mathbb{R})$. In conclusion, with $\mathcal{H}$ being the Filipovic space, we choose a 
different space for the noise $L$ to open up for flexibility in modelling the spatial correlation, and an integral operator for $\Psi$ to ensure that we map the noise into the Filipovic space, at the same time modeling the Samuelson effect.

To follow up on the integral operator, we know the function $\widetilde{\sigma}(x, y)$ on the diagonal $x=y$, since here we want to match with the observed curve for the Samuelson effect. In a neighborhood around $x$, we smoothly interpolate to zero such that $\widetilde{\sigma}(x, \cdot)$ has a support close to $\{x\}$, and such that the function defines an integral operator being sufficiently regular. One possibility is to define $\tilde{\sigma}(x, y)=\eta(x) \bar{\sigma}(|x-y|)$, where $\bar{\sigma}: \mathbb{R}_{+} \rightarrow \mathbb{R}_{+}$is smooth, $\bar{\sigma}(0)=1$, and $\operatorname{supp} \bar{\sigma}$ is the interval $(-a, a)$ for $a$ small. With this definition, we have that $\eta$ models the Samuelson effect, the operator $\Psi$ is a convolution product with $\bar{\sigma}$, followed by a multiplication with $\eta$. With $\eta$ being an element of the Filipovic space, we have specified $\Psi$ as desired. By inspection of the curve for the volatility term structure in Figure 4, a first-order approximation of it could be a function $\eta(x)=a \exp (-\zeta x)+b$, for constants $a, \zeta$ and $b$, where $b>0$ is the long-term level and $\zeta>0$ measures the exponential decay in the short end. We note that $\eta(0)=a+b$ will be the spot volatility. With such a specification, $\eta$ will be an element of the Filipovic space since it is smooth and asymptotically constant. As we see, this simple model fails to account for the pronounced bumps in the curve that we have discussed earlier. By a more sophisticated model, one can take these into account as well.

Our empirical analysis also show indications of stochastic volatility effects. We will not discuss possible GARCH/ARCH specifications in continuous time, but briefly just mention that we can choose $\Sigma_{s}=V_{s} \Psi$, where $s \mapsto V_{s}$ is a $\mathbb{R}_{+}$-valued stochastic process. For example, we can define $V$ to be the Heston stochastic volatility dynamics (see Heston (1993)) or the BNS stochastic volatility model (see Barndorff-Nielsen and Shephard (2001)). In this case, it would be natural to suppose $L$ to be a Wiener process in $L^{2}(\mathbb{R})$, since the additional stochastic volatility process $V$ will induce non-Gaussian distributed residuals. We leave the further discussion on stochastic volatility models in infinite di- 
mensional term structure models for future research (see however, Benth, Rüdiger, and Süss (2015) for a Hilbert-valued Ornstein-Uhlenbeck processes with stochastic volatility).

\section{Conclusion and future work}

In this study, we derived a spatio-temporal dynamical model based on the Heath-JarrowMorton (HJM) approach under the Musiela parametrization (see Heath, Jarrow, and Morton (1992)), which ensures an arbitrage-free model for electricity forward prices. A discretized version of the model has been fitted to electricity forward prices to examine the probabilistic characteristics of the data. We disentangled the seasonal pattern from the market price of risk and random perturbations of prices and analysed empirically their statistical properties.

As a special feature of our model, we further disentangled the temporal from spatial (time to maturity) effects on the dynamics of forward prices, which marks one of the main contributions of this study to the academic literature (see Andresen, Koekebakker, and Westgaard (2010)). After filtering out both temporal and spatial effects of price forward curves and the market price of risk, we estimated the term structure volatility. Finally, our model residuals show a white-noise pattern, which validates our modeling assumptions.

The model has been fitted to a unique data set of historical daily PFCs for the German electricity market. We firstly performed a deseasonalization of the initial curves, where the seasonal component takes into account typical deterministic dynamics observed in the German electricity prices (see Paraschiv, Fleten, and Schürle (2015), Paraschiv (2013)). We further estimated the risk premia in the deseasonalized curves (stochastic component) and examined, in this context, the distribution of the noise: term structure volatility and its spatio-temporal correlations structures. Our results show that the shortterm risk premia oscillate around zero, but become negative in the long run, which is consistent with the empirical literature (Paraschiv, Fleten, and Schürle (2015), Burger, 
Graeber, and Schindlmayr (2007)). We found that the noise marginals are coloured-noise with a strong leptokurtic pattern and heavy-tails, which have been successfully modeled by a normal inverse Gaussian distribution (NIG). There were signs of stochastic volatility effects as well. The high performance of the NIG distribution in modeling the noise marginals of forward electricity prices confirms previous findings of Frestad, Benth, and Koekebakker (2010). The term structure of volatility decays overall with increasing time to maturity, a typical Samuelson effect. However, the term structure of volatility in our data set has additionally clear bumps around the maturity of 1 month and third quarter, both being related to an increased activity in the market for the corresponding futures contracts. Our analysis also detects a fast decaying pattern in the spatial correlations as a function of distance between times to maturity.

Our empirical findings mark an additional contribution over existing related literature Andresen, Koekebakker, and Westgaard (2010): we shed light on the statistical properties of risk premia, of the noise, volatility term structure and of the spatio-temporal noise correlation structures. Notably, we look at price residuals where the maturity effect is corrected for, unlike the approach of Andresen, Koekebakker, and Westgaard (2010).

Based on the empirical insights, we revisited the spatio-temporal model of forward prices and derived a mathematical model for the noise. After explaining the Samuelson effect in the volatility term structure, the residuals are modeled by an infinite dimensional NIG Lévy process, which allows for a natural formulation of a covariance functional. We model, in this way, the typical fat tails and fast-decaying pattern of spatial correlations. Still, our empirical findings show some slight remaining volatility clustering effects in the standardized residuals, which can be described by a stochastic volatility model formulation. However, we will discuss and develop stochastic volatility models in infinite dimensional term structure models in future research. 


\section{Acknowledgements}

Fred Espen Benth acknowledges financial support from the project FINEWSTOCH, funded by the Norwegian Research Council. The authors thank very much Gido Haarbrücker, Claus Liebenberger and Michael Schürle from the Institute for Operations Research and Computational Finance, University of St. Gallen, for the continued support in the data collection step of this work. We are grateful to the reviewers for constructive feedback on our paper, resulting in a significant improvement in the presentation of our analysis.

\section{References}

Andresen, A., S. Koekebakker, and S. Westgaard (2010): "Modeling electricity forward prices using the multivariate normal inverse Gaussian distribution," The Journal of Energy Markets, 3(3), 3-25.

Audet, A., P. Heiskanen, J. Keppo, and A. Vehviläinen (2004): "Modeling electricity forward curve dynamics in the Nordic market," in Modelling Prices in Competitive Electricity Markets, ed. by D. W. Bunn, pp. 251-265. Wiley, Chichester.

Barndorff-Nielsen, O. E., F. E. Benth, and A. Veraart (2013): "Modelling energy spot prices by volatility modulated Lévy-driven Volterra processes," Bernoulli, $19,803-845$.

(2014): "Modelling electricity futures by ambit fields," Advances in Applied Probability, 46, 719-745.

(2015): "Cross-commodity modelling by multivariate ambit fields," in Commodities, Energy and Environmental Finance, Fields Institute Communications Series, ed. by R. Aid, M. Ludkovski, and R. Sircar, pp. 109-148. Springer Verlag.

Barndorff-Nielsen, O. E., And N. Shephard (2001): "Non-Gaussian OrnsteinUhlenbeck-based models and some of their uses in economics," Journal of the Royal Statistical Society, Series B, 63, 167-241.

BARTh, A., And F. E. Benth (2014): "The forward dynamics in energy markets infinite dimensional modeling and simulation," Stochastics, 86, 932-966.

Benth, F.-E., J. Kallsen, and T. Meyer-Brandis (2007): "A non-Gaussian OrnsteinUhlenbeck process for electricity spot price modeling and derivatives pricing," $A p$ plied Mathematical Finance, 14, 153-169. 
Benth, F. E., C. Klüppelberg, G. Müller, and L. Vos (2014): "Futures pricing in electricity markets based on stable CARMA spot models," Energy Economics, 44, $392-406$.

Benth, F. E., And S. Koekebakker (2008): "Stochastic modeling of financial electricity contracts," Energy Economics, 30, 1116-1157.

Benth, F. E., S. Koekebakker, and F. Ollmar (2007): "Extracting and applying smooth forward curves from average-based commodity contracts with seasonal variation," Journal of Derivatives, 15, 52-66.

Benth, F. E., And P. KrüHner (2014): "Representation of infinite-dimensional forward price models in commodity markets," Communications in Mathematics and Statistics, 2, 47-106.

(2015): "Derivatives pricing in energy markets: an infinite dimensional approach," SIAM Journal on Financial Mathematics, 6, 825-869.

Benth, F. E., And P. Krühner (2015): "Subordination of Hilbert space valued Lévy processes," Stochastics, 87, 458-476.

Benth, F. E., And J. Lempa (2014): "Optimal portfolios in commodity markets," Finance 8 Stochastics, 18, 407-430.

Benth, F. E., B. Rüdiger, And A. Süss (2015): "Ornstein-Uhlenbeck processes in Hilbert space with non-Gaussian stochastic volatility," Submitted manuscript. Available on arXiv:1506.07245.

Benth, F. E., J. Šaltytė Benth, and S. Koekebakker (2008): Stochastic Modelling of Electricity and Related Markets. World Scientific, Singapore.

Bloechlinger, L. (2008): Power prices - a regime-switching spot/forward price model with Kim filter estimation, Dissertation of the University of St. Gallen, No. 3442.

Burger, M., B. Graeber, and G. Schindlmayr (2007): Managing Energy Risk An Integrated View on Power and Other Energy Markets. Wiley.

Caldana, R., G. Fusai, and A. Roncoroni (2016): "Energy forward curves with thin granularity," Available at SSRN: abstract=2777990.

Cartea, A., and M. Figueroa (2005): "Pricing in electricity markets: a mean reverting jump diffusion model with seasonality," Applied Mathematical Finance, 12, 313-335. 
Clewlow, L., and C. Strickland (2000): Energy Derivatives: Pricing and Risk Management. Lacima Publications, London.

Filipovic, D. (2001): Consistency Problems for Heath-Jarrow-Morton Interest Rate Models. Springer Verlag, Berlin Heidelberg.

Fleten, S.-E., and J. Lemming (2003): "Constructing forward price curves in electricity markets," Energy Economics, 25, 409-424.

Folland, G. (1984): Real Analysis. John Wiley \& Sons, New York.

Frestad, D. (2008): "Common and unique factors influencing daily swap returns in the Nordic electricity market, 1997-2005," Energy Economics, 30, 1081-1097.

Frestad, D., F. Benth, and S. Koekebakker (2010): "Modeling term structure dynamics in the Nordic electricity swap market," Energy Journal, 31, 53-86.

Garcia, I., C. KlüppelberG, and G. Müller (2011): "Estimation of Stable CARMA Models with an Application to Electricity Spot Prices," Statistical Modelling, 11, 447470.

Geman, H. (2005): Commodities and Commodity Derivatives: Modeling and Pricing for Agriculturals, Metals and Energy, The Wiley Finance Series. John Wiley and Sons.

Goldstein, R. S. (2000): "The Term Structure of Interest Rates as a Random Field," Review of Financial Studies, 13, 365-384.

Heath, D., R. Jarrow, and A. Morton (1992): "Bond pricing and the term structure of interest rates: a new methodology for contingent claim valuation," Econometrica, $60,77-105$.

Heston, S. (1993): "A closed-form solution for options with stochastic volatility with applications to bond and currency options," Review of Financial Studies, 6, 327-343.

Kennedy, D. P. (1994): "The term structure of interest rates as a Gaussian random field," Mathematical Finance, 4, 247-258.

Kiesel, R., G. Schindlmayr, and R. Boerger (2009): "A two-factor model for the electricity forward market," Quantitative Finance, 9, 279-287.

Koekebakker, S., and F. Ollmar (2005): "Forward curve dynamics in the Nordic electricity market," Managerial Finance, 31, 73-94. 
Lucia, J. J., AND E. S. Schwartz (2002): "Electricity prices and power derivatives: evidence from the Nordic power exchange," Review of Derivatives Research, 5, 5-50.

Paraschiv, F. (2013): "Price dynamics in electricity markets," in Risk Management in Energy Production and Trading, ed. by P. G. Kovacevic, R.M., and M. Vespucci, pp. 57-111. Springer.

Paraschiv, F., D. Erni, and R. Pietsch (2014): "The impact of renewable energies on EEX day-ahead electricity prices," Energy Policy, 73, 196-210.

Paraschiv, F., S.-E. Fleten, and M. Schürle (2015): "A spot-forward model for electricity prices with regime shifts," Energy Economics, 47, 142-153.

Perron, P., And S. NG (1996): "Useful modiffications to some unit root tests with dependent errors and their local asymptotic properties," Review of Economic Studies, $63,435-463$.

Peszat, S., And J. ZabcZyk (2007): Stochastic Partial Differential Equations with Lévy Noise. Cambridge University Press, Cambridge.

PIETZ, M. (2009): "Risk premia in electricity wholesale spot markets empirical evidence from Germany," Working Paper, Center for Entrepreneurial and Financial Studies. Technical University of Munich.

Roncoroni, A., and H. Geman (2006): "Understanding the fine structure of electricity prices," Journal of Business, 79, 1225-1261.

Roncoroni, A., And P. Guiotto (2001): "Theory and Calibration of HJM with Shape Factors," Mathematical Finance, Springer Finance, pp. 40\%-426.

Weron, R., AND S. BoraK (2008): "A semiparametric factor model for electricity forward curve dynamics," Journal of Energy Markets, 1, 3-16.

Weron, R., AND M. Zator (2014): "Revisiting the relationship between spot and futures prices in the Nord Pool electricity market," Energy Economics, 44, 178-190.

Wright, J. H. (1999): "Testing for a unit root in the volatility of asset returns," Journal of applied econometrics, 14, 309-318. 